\title{
HIERARCHICAL MODEL REDUCTION DRIVEN BY A PROPER ORTHOGONAL DECOMPOSITION FOR PARAMETRIZED ADVECTION-DIFFUSION-REACTION PROBLEMS*
}

\author{
MASSIMILIANO LUPO PASINI ${ }^{\dagger}$ AND SIMONA PEROTTO ${ }^{\ddagger}$
}

\begin{abstract}
This work combines the Hierarchical Model (HiMod) reduction technique with a standard Proper Orthogonal Decomposition (POD) to solve parametrized partial differential equations for the modeling of advectiondiffusion-reaction phenomena in elongated domains (e.g., pipes). This combination leads to what we define as HiPOD model reduction, which merges the reliability of HiMod reduction with the computational efficiency of POD. Two HiPOD techniques are presented and assessed by an extensive numerical verification.
\end{abstract}

Key words. hierarchical model reduction, proper orthogonal decomposition, parametric partial differential equations, finite elements, spectral methods

AMS subject classifications. $65 \mathrm{~N} 30,65 \mathrm{~N} 35,65 \mathrm{~T} 40$

1. Motivations. Parametrized partial differential equations (PDEs) arise in several contexts such as inverse problems, control, optimization, uncertainty quantification, and risk assessment. In most of these applications, the number of parameters may become very large so that an efficient numerical approximation of parametric PDEs represents a challenging computational issue; see, e.g., [2, 3, 6, 7]. Parametric model order reduction aims at reducing the computational effort associated with a parametric modeling, for instance, in many-query and real-time tasks, where the occurrence of the curse of dimensionality raises the necessity to propose numerical methods to sustain the computational cost.

Many of the model reduction techniques currently employed in engineering practice exploit the offline/online paradigm to efficiently reduce the numerical effort. This is the case, for instance, for the well-known reduced basis method [17, 33], where during the offline phase, a reduced basis is precomputed by solving a high-fidelity model (the "truth") for certain samples of the parameter, while in the online phase, the reduced model is evaluated to predict a new scenario (i.e., for a value of the parameter not previously sampled). From a practical viewpoint, the offline stage remains the bottleneck of an offline/online decomposition, especially when many samples are needed like for multiparametric problems.

To tackle this issue, we propose to replace the "truth" with a reduced-order model that exhibits a high accuracy although it is characterized by a contained computational demand. For this purpose, we employ the reduced solution provided by a Hierarchical Model (HiMod) discretization [12, 26, 28, 31] as high-fidelity model.

HiMod reduction proved to be an effective tool to model partial differential equations characterized by a privileged dynamics aligned with the dominant dimension of the domain (e.g., flows of fluid in channels, pipes, or vessels), which may be locally modified by a secondary dynamics evolving along the transverse sections $[8,15,29]$. Analogously to other model

*Received May 3, 2021. Accepted November 11, 2021. Published online on December 17, 2021. Recommended by Roland Herzog. This manuscript has been authored in part by UT-Battelle, LLC, under contract DE-AC0500OR22725 with the US Department of Energy (DOE). The US government retains and the publisher, by accepting the article for publication, acknowledges that the US government retains a nonexclusive, paid-up, irrevocable, worldwide license to publish or reproduce the published form of this manuscript, or allow others to do so, for US government purposes. DOE will provide public access to these results of federally sponsored research in accordance with the DOE Public Access Plan (http://energy.gov/downloads/doe-public-access-plan).

${ }^{\dagger}$ Computational Sciences and Engineering Division, Oak Ridge National Laboratory, 1 Bethel Valley Road, Oak Ridge, TN, USA, 37831 (lupopasinimeornl.gov).

${ }^{\ddagger}$ MOX-Dipartimento di Matematica, Politecnico di Milano, Piazza L. da Vinci 32, I-20133 Milano, Italy (simona.perotto@polimi.it). 
reduction procedures $[9,10,14,16,24,34]$, a HiMod discretization starts from a standard separation of variables and approximates the mainstream and the secondary dynamics by means of different numerical methods. In the seminal papers, the main direction of the flux is discretized by one-dimensional (1D) finite elements, while the transverse dynamics are recovered by using few degrees of freedom via a suitable modal basis. This separate discretization yields a system of coupled 1D problems whose coefficients include the effect of the transverse dynamics. The reliability exhibited by HiMod is considerably higher compared with standard $1 \mathrm{D}$ reduced models, whereas the computational effort remains absolutely affordable. Indeed, HiMod reduction is characterized by a linear dependence of the computational cost on the number of degrees of freedom, in contrast to a standard finite element model, which requires a suitable power of such a number.

In this paper, we focus on two different ways to combine a Proper Orthogonal Decomposition (POD) [19, 20, 21, 22, 36] with HiMod reduction, setting what we define as HiPOD model reduction. The first approach is very straightforward, and it has been introduced in [4]. The second variant, which represents the actual novelty of the paper, is more complex and takes advantage of the separation of variables implied by a HiMod approximation. Independently of the adopted procedure, the HiMod discretization significantly reduces the computational effort of the offline phase without compromising its reliability. At the same time, the online phase relies on the efficiency of a POD formulation so that a system of very small dimensionality is solved to approximate the parametric problem at hand.

From a different viewpoint, we can conceive HiPOD as a new method to construct HiMod approximations that differ from the classical approach proposed in $[12,28]$. The HiMod approximation is now built by resorting to a reduced basis generated by a data-driven procedure. This choice significantly lowers the computational costs without compromising the quality of the reduced solution.

The paper is organized as follows. Section 2 applies the HiMod discretization to reference parametric advection-diffusion-reaction problems and numerically assesses the reliability of the high-fidelity model. Section 3 introduces the two HiPOD model reduction procedures and provides an extensive numerical verification to investigate the robustness of the proposed approaches with respect to the truncation of the POD basis, the extrapolation, and the possibility to explore multi-parametric settings. In Section 4 we look for possible settings where one of the two HiPOD approximations outperforms the other. Finally, some conclusions are drawn in the last section, and possible future developments of the current work are provided.

2. HiMod reduction: the basics. HiMod reduction is performed under the specific assumption that the computational domain $\Omega \subset \mathbb{R}^{d}$, with $d=2,3$, can be expressed as a Cartesian product, $\bigcup_{x \in \Omega_{1 D}}\{x\} \times \Sigma_{x}$, where $\Omega_{1 D}$ is a 1D horizontal supporting fiber, while $\Sigma_{x} \subset \mathbb{R}^{d-1}$ denotes the transverse section at the generic point $x$ along $\Omega_{1 D}[12,26,28,31]$. The reference geometry is a pipe, where the dominant dynamic is parallel to $\Omega_{1 D}$, whereas the transverse dynamics occur along the fibers $\Sigma_{x}$. For the sake of simplicity, we select $\Omega_{1 D} \equiv(a, b) \subset \mathbb{R}$. For the general case where $\Omega_{1 D}$ coincides with a bent centerline, we refer to $[8,25,29]$. Then, via an invertible map $\Psi: \Omega \rightarrow \widehat{\Omega}$, we change the physical domain $\Omega$ into a reference domain $\widehat{\Omega}=\Omega_{1 D} \times \widehat{\Sigma}$, which shares the same supporting fiber as in $\Omega$ and where $\widehat{\Sigma} \subset \mathbb{R}^{d-1}$ denotes the reference fiber. In particular, for any point $\mathbf{z}=(x, \mathbf{y}) \in \Omega$, there exists a point $\widehat{\mathbf{z}}=(\widehat{x}, \widehat{\mathbf{y}}) \in \widehat{\Omega}$ such that $\widehat{\mathbf{z}}=\Psi(\mathbf{z})$, with $\widehat{x} \equiv x$ and $\widehat{\mathbf{y}}=\psi_{x}(\mathbf{y})$, where $\psi_{x}: \Sigma_{x} \rightarrow \widehat{\Sigma}$ is the map between the generic and the reference transverse fiber. Hereafter, we assume $\psi_{x}$ to be a $C^{1}$-diffeomorphism for all $x \in \Omega_{1 D}$ and $\Psi$ to be differentiable with respect to $\mathbf{z}$. The reference domain $\widehat{\Omega}$ represents the setting where the computations are actually performed and where all the constants can be explicitly computed. More details about the maps $\Psi$ and $\psi_{x}$ are available in [28]. 


\section{ETNA}

Kent State University and

Johann Radon Institute (RICAM)

As a reference problem, we choose a parametrized elliptic PDE defined on $\Omega$, which can be recast into the following weak form: given the parameter $\alpha \in \mathcal{P}$,

$$
\text { find } u(\alpha) \in V \quad \text { s.t. } \quad a(u(\alpha), v ; \alpha)=f(v ; \alpha) \quad \forall v \in V,
$$

where $\mathcal{P} \subset \mathbb{R}^{p}$ is the set of admissible parameters, $V \subseteq H^{1}(\Omega)$ is a Hilbert space depending on the PDE problem and on the selected boundary conditions with the standard notation for function spaces [11], $a(\cdot, \cdot ; \alpha): V \times V \times \mathcal{P} \rightarrow \mathbb{R}$ and $f(\cdot ; \alpha): V \times \mathcal{P} \rightarrow \mathbb{R}$ denote a parametrized bilinear and linear form, respectively, where the linearity property holds with respect to all the variables but $\alpha$. Suitable hypotheses are imposed on the problem data to guarantee the well-posedness of the formulation (2.1) for any $\alpha \in \mathcal{P}$. Moreover, we assume an affine parameter dependence [17, 33].

We focus on a scalar linear advection-diffusion-reaction (ADR) problem completed for the sake of simplicity with full homogeneous Dirichlet boundary conditions so that the bilinear and the linear forms in (2.1) are

$$
a(w, z ; \alpha)=\int_{\Omega} \mu \nabla w \cdot \nabla z d \Omega+\int_{\Omega}(\mathbf{b} \cdot \nabla w+\sigma w) z d \Omega, \quad f(z ; \alpha)=\int_{\Omega} f z d \Omega
$$

with $w, z \in V=H_{0}^{1}(\Omega)$. The parameter $\alpha$ coincides with one or several of the problem data, chosen among the viscosity $\mu$, the advective field $\mathbf{b}=\left[b_{1}, \ldots, b_{d}\right]^{T}$, the reaction coefficient $\sigma$, the source term $f$, or a boundary value when other boundary conditions more general than homogeneous Dirichlet data are assigned.

HiMod reduction performs a different discretization along the supporting and the transverse directions. For this purpose, we introduce a $1 \mathrm{D}$ discrete space $V_{1 D} \subset H_{0}^{1}\left(\Omega_{1 D}\right)$, with $\operatorname{dim}\left(V_{1 D}\right)=N_{h}<+\infty$, of functions vanishing at $a$ and $b$ and a modal basis $\left\{\varphi_{k}\right\}_{k \in \mathbb{N}^{+}}$of functions defined on $\widehat{\Sigma}$ that are orthonormal with respect to the $L^{2}(\widehat{\Sigma})$-scalar product and which satisfy the data assigned on $\Gamma_{L}=\bigcup_{x \in \Omega_{1 D}} \partial \Sigma_{x}$. For further details about the choice of the modal basis also in the presence of general boundary data on $\Gamma_{L}$, we refer to $[1,15,28]$. Concerning $V_{1 D}$, a standard choice is the finite element space [12, 26, 28, 30, 31, 32] or an isogeometric discretization when $\Omega$ is not rectilinear $[8,29]$. Thus, the HiMod reduced space can be defined as

$$
V_{m}=\left\{v_{m}(x, \mathbf{y} ; \alpha)=\sum_{k=1}^{m} \sum_{j=1}^{N_{h}} \widetilde{v}_{k, j}^{\alpha} \vartheta_{j}(x) \varphi_{k}\left(\psi_{x}(\mathbf{y})\right), \text { for } x \in \Omega_{1 D}, \mathbf{y} \in \Sigma_{x}, \alpha \in \mathcal{P}\right\},
$$

with $\left\{\vartheta_{j}\right\}_{j=1}^{N_{h}}$ being a basis for the space $V_{1 D}$ so that $\widetilde{v}_{k}(x ; \alpha)=\sum_{j=1}^{N_{h}} \widetilde{v}_{k, j}^{\alpha} \vartheta_{j}(x) \in V_{1 D}$ denotes the frequency coefficient of $v_{m}$ associated with the $k$ th modal function $\varphi_{k}$.

The modal index $m \in \mathbb{N}^{+}$establishes the level of detail of the HiMod approximation in the hierarchy of reduced spaces $\left\{V_{m}\right\}_{m}$. This index is selected by the user through some preliminary (geometric or physical) information about the problem at hand or via an automatic procedure based on an a posteriori modeling error analysis [30,32]. Additionally, the index $m$ can be the same in the whole $\Omega$, or it can be locally tuned along the domain to match possible heterogeneities of the solution. We refer the interested reader to [26, 31], where a survey about the different criteria to choose $m$ is provided.

The HiMod approximation to problem (2.1) becomes

(2.3) find $u_{m}(\alpha)=u_{m}(x, \mathbf{y} ; \alpha) \in V_{m} \quad$ s.t. $\quad a\left(u_{m}(\alpha), v_{m} ; \alpha\right)=f\left(v_{m} ; \alpha\right) \quad \forall v_{m} \in V_{m}$,

for a given parameter $\alpha \in \mathcal{P}$ and for a selected modal index $m \in \mathbb{N}^{+}$. Following [28], we add a conformity and a spectral approximability assumption on the HiMod space $V_{m}$ to ensure the 
well-posedness of the formulation (2.3) along with a standard density assumption on the space $V_{1 D}$ to guarantee the convergence of the HiMod approximation $u_{m}(\alpha)$ to the full solution $u(\alpha)$ in (2.1). From a computational viewpoint, after applying the HiMod expansion to the solution $u_{m}(\alpha)$ in (2.3) and choosing the test function $v_{m}$ as the generic product $\vartheta_{t} \varphi_{q}$, with $q=1, \ldots, m$ and $t=1, \ldots, N_{h}$, the HiMod formulation turns into the system

$$
A_{m}(\alpha) \mathbf{u}_{m}(\alpha)=\mathbf{f}_{m}(\alpha)
$$

of $m$ 1D coupled problems, where $A_{m}(\alpha) \in \mathbb{R}^{m N_{h} \times m N_{h}}$ and $\mathbf{f}_{m}(\alpha) \in \mathbb{R}^{m N_{h}}$ are the HiMod stiffness matrix and right-hand side, while

$$
\mathbf{u}_{m}(\alpha)=\left[\widetilde{u}_{1,1}^{\alpha}, \ldots, \widetilde{u}_{1, N_{h}}^{\alpha}, \widetilde{u}_{2,1}^{\alpha}, \ldots, \widetilde{u}_{2, N_{h}}^{\alpha}, \ldots, \widetilde{u}_{m, 1}^{\alpha}, \ldots, \widetilde{u}_{m, N_{h}}^{\alpha}\right]^{T} \in \mathbb{R}^{m N_{h}}
$$

is the vector describing the solution,

$$
u_{m}(x, \mathbf{y} ; \alpha)=\sum_{k=1}^{m} \sum_{j=1}^{N_{h}} \widetilde{u}_{k, j}^{\alpha} \vartheta_{j}(x) \varphi_{k}\left(\psi_{x}(\mathbf{y})\right),
$$

discretized via the HiMod approach, where $\left\{\widetilde{u}_{k, j}^{\alpha}\right\}_{k=1, j=1}^{m, N_{h}}$ are the modal coefficients; see [12, 28] for additional computational details.

When the mainstream dominates the transverse dynamics (i.e., for small values of $m$ ), the HiMod procedure has been shown to considerably reduce the computational burden associated with a standard discretization of problem (2.1) without affecting the accuracy of the simulation $[8,15,23]$.

2.1. Reliability check of the HiMod reduction. The numerical assessment of this paper focuses on the two-dimensional (2D) setting. In this section, we qualitatively investigate the reliability of the HiMod reduction for two ADR problems completed with different boundary conditions, and we disregard the role played by the PDE parameters at this stage. For the HiMod discretization, we resort to linear finite elements (FE) along $\Omega_{1 D}$, whereas we describe the transverse dynamics by a sinusoidal modal basis. For a quantitative analysis as well as for a three-dimensional (3D) verification of the HiMod approximation, we refer the reader to $[1,8,15,28]$.

2.1.1. Test case 1. We define the domain $\Omega$ as the rectangle $(0,3) \times(0,1)$, while the problem data in (2.2) are

$$
\mu(x, y)=1, \quad \mathbf{b}(x, y)=[3,0]^{T}, \quad \sigma(x, y)=0, \quad f(x, y)=1-2 x+3 y .
$$

The image at the top of Figure 2.1 displays the reference (full) solution computed with linear finite elements on a uniform unstructured grid of 260058 triangles. The chosen data justify the diffusive trend of the solution, which alternates a maximum to a minimum area.

With regards to the HiMod approximation, we subdivide the supporting fiber $[0,3]$ into 60 uniform subintervals, and we discretize the transverse dynamics by gradually increasing the number $m$ of modal basis functions. The bottom panels in Figure 2.1 display the HiMod approximations for $m=1$ (left) and $m=2$ (right). It is evident that two modes are enough to ensure a qualitatively good accuracy of the reduced solution with a considerable reduction in terms of the degrees of freedom (dofs) (120 dofs for the HiMod approximation to be compared with 373464 dofs for the finite element model). 

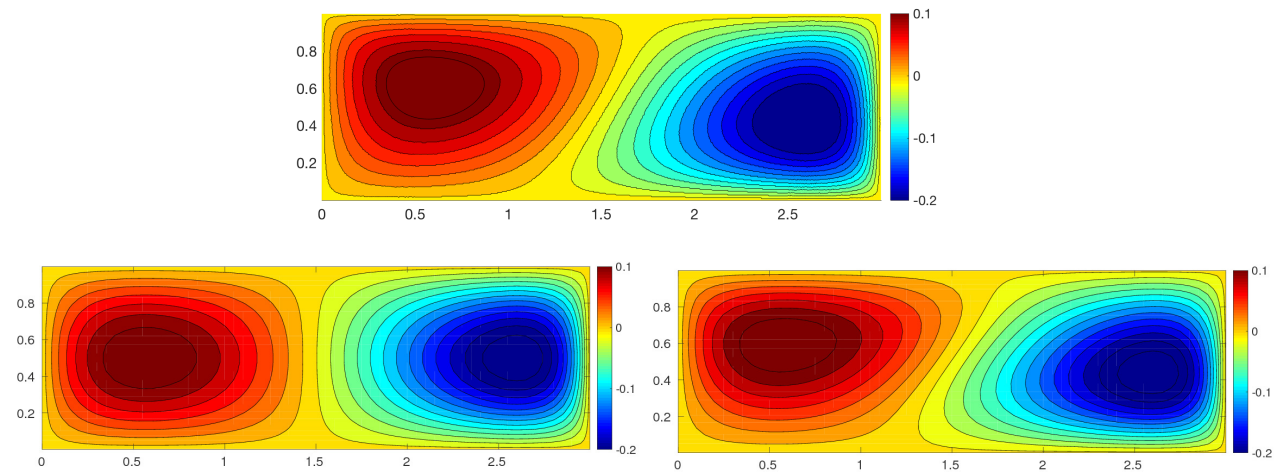

FIG. 2.1. HiMod verification (test case 1): reference FE (top) and HiMod (bottom) solution for $m=1$ (left) and $m=2$ (right).

2.1.2. Test case 2. The domain $\Omega$ is now taken as the rectangle $(0,6) \times(0,1)$, and we select the problem data as

$$
\begin{aligned}
& \mu(x, y)=0.24, \quad \mathbf{b}(x, y)=[5, \sin (6 x)]^{T}, \quad \sigma(x, y)=0.1, \\
& f(x, y)=10 \chi_{C_{1}}(x, y)+10 \chi_{C_{2}}(x, y),
\end{aligned}
$$

where $\chi_{\omega}$ denotes the characteristic function associated with the generic region $\omega \subset \mathbb{R}^{2}$, while $C_{1}$ and $C_{2}$ identify the ellipsoidal areas $\left\{(x, y):(x-0.75)^{2}+0.4(y-0.25)^{2}<0.01\right\}$ and $\left\{(x, y):(x-0.75)^{2}+0.4(y-0.75)^{2}<0.01\right\}$, respectively. The ADR problem is completed with homogeneous Neumann data on $\Gamma_{N}=\{(x, y): x=6,0 \leq y \leq 1\}$ and with homogeneous Dirichlet condition on $\Gamma_{D}=\partial \Omega \backslash \Gamma_{N}$, so that $V \equiv H_{\Gamma_{D}}^{1}(\Omega)$ in (2.1). The top panel of Figure 2.2 displays the contour plot of the approximation obtained with linear finite elements on a uniform and unstructured mesh consisting of 3200 elements. We draw the attention of the reader to the oscillatory dynamics induced by the sinusoidal field and the presence of the two localized sources in $C_{1}$ and $C_{2}$. Moreover, no stabilization is applied despite the convection overcomes the diffusion. HiMod reduction is applied by introducing a uniform subdivision of $\Omega_{1 D}$ into 120 subintervals and by employing an increasing number of modes. We do not introduce any stabilization also for the HiMod discretization. Figure 2.2, second to fourth row, provides the HiMod approximation for $m=2, m=3$, and $m=5$, respectively. At least five modes have to be employed to obtain a qualitatively reliable HiMod solution. As expected, the number of HiMod dofs is considerably lower compared with the finite element case (600 versus 305171 dofs).

3. HiPOD techniques. The goal of the HiPOD techniques is to build a HiMod approximation for problem (2.1) at a computational cost lower than that for the HiMod system (2.4). For this purpose, we resort to a POD approach by adopting the offline/online paradigm $[19,20,21,22,36]$. In particular, during the offline phase, we discretize the problem (2.1) via HiMod for different choices of $\alpha$ to extract the POD (reduced) basis. In the online phase, we employ such a basis to approximate the HiMod solution of (2.1) for a value $\alpha=\alpha^{*}$ of the parameter not yet sampled.

In this paper we explore two different HiPOD approaches. The first one is the most straightforward procedure, where the online phase is carried out by resorting to a standard projection [4]. In the second approach, we drive the online phase by means of interpolation, following [37]. This second variant takes advantage of the separation of variables implied by a HiMod discretization. 

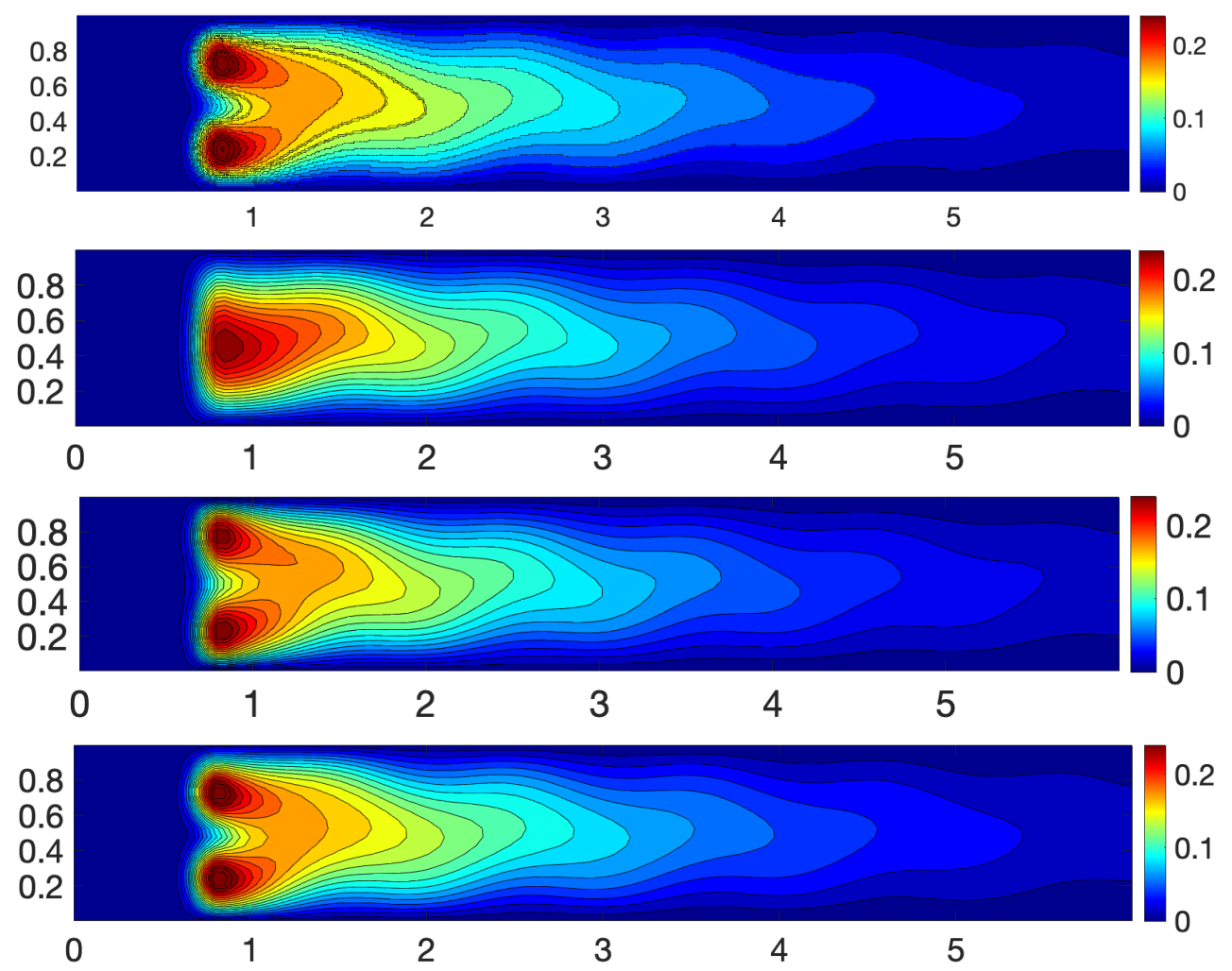

FIG. 2.2. HiMod verification (test case 2): reference FE (first row) and HiMod solution for $m=2$ (second row), $m=3$ (third row), and $m=5$ (fourth row).

The leading feature of a HiPOD technique is that it contains the typical computational burden of an offline phase. Actually, the POD is applied to solutions which have already been reduced via HiMod in contrast to standard approaches where full solutions (e.g., finite element approximations) are employed to sample the phenomenon at hand. Finally, we notice that HiPOD methods are fully general since, a priori, any model reduction technique may replace the HiMod discretization during the offline phase.

3.1. The basic HiPOD approach. We start the offline phase by assembling the snapshot (or response) matrix $S$. To this aim, we select $p$ different values $\alpha_{i}$ of the parameter $\alpha$, and we compute the HiMod approximation of the associated problem $(2.1)$, for $i=1, \ldots, p$. We employ the same discretization along $\Omega_{1 D}$ and the same modal expansion for the transverse dynamics so that, according to the representation (2.5), each HiMod solution is identified by the $m N_{h}$ coefficients $\left\{\widetilde{u}_{k, j}^{\alpha_{i}}\right\}_{k=1, j=1}^{m, N_{h}}$ or, likewise, by the vector

$$
\mathbf{u}_{m}\left(\alpha_{i}\right)=[\underbrace{\widetilde{u}_{1,1}^{\alpha_{i}}, \ldots, \widetilde{u}_{1, N_{h}}^{\alpha_{i}}}_{k=1}, \underbrace{\widetilde{u}_{2,1}^{\alpha_{i}}, \ldots, \widetilde{u}_{2, N_{h}}^{\alpha_{i}}}_{k=2}, \ldots, \underbrace{\widetilde{u}_{m, 1}^{\alpha_{i}}, \ldots, \widetilde{u}_{m, N_{h}}^{\alpha_{i}}}_{k=m}]^{T} \in \mathbb{R}^{m N_{h}},
$$

collecting the modal coefficients by mode. Thus, we assemble the snapshot matrix

$$
S=\left[\mathbf{u}_{m}\left(\alpha_{1}\right), \mathbf{u}_{m}\left(\alpha_{2}\right), \ldots, \mathbf{u}_{m}\left(\alpha_{p}\right)\right] \in \mathbb{R}^{\left(m N_{h}\right) \times p},
$$


and the matrix

$$
\mathcal{V}=S-\frac{1}{p} \sum_{i=1}^{p}\left[\mathbf{u}_{m}\left(\alpha_{i}\right), \mathbf{u}_{m}\left(\alpha_{i}\right), \ldots, \mathbf{u}_{m}\left(\alpha_{i}\right)\right] \in \mathbb{R}^{\left(m N_{h}\right) \times p},
$$

which is characterized by having zero average. The matrix $\mathcal{V}$ is the array actually employed to extract the POD basis. For this purpose, we apply the Singular Value Decomposition (SVD) to $\mathcal{V}$ to obtain

$$
\mathcal{V}=\Phi \Sigma \Psi^{T}
$$

where $\Phi \in \mathbb{R}^{\left(m N_{h}\right) \times\left(m N_{h}\right)}$ and $\Psi \in \mathbb{R}^{p \times p}$ are the unitary matrices gathering the left and the right singular vectors of $\mathcal{V}$, while $\Sigma=\operatorname{diag}\left(\sigma_{1}, \ldots, \sigma_{\gamma}\right) \in \mathbb{R}^{\left(m N_{h}\right) \times p}$ is the pseudo-diagonal matrix of the singular values of $\mathcal{V}$ with $\sigma_{1} \geq \sigma_{2} \geq \cdots \geq \sigma_{\gamma} \geq 0$ and $\gamma=\min \left(m N_{h}, p\right)$ [13]. In the numerical assessment below, we always assume $\gamma=p$.

The decomposition (3.3) allows us to define the POD orthogonal reduced basis, being the set of the first $l$ most significant left singular vectors $\left\{\phi_{i}\right\}_{i=1}^{l}$ of $\mathcal{V}$ so that the reduced POD space is $V_{\mathrm{POD}}^{l}=\operatorname{span}\left\{\phi_{1}, \ldots, \phi_{l}\right\}$ with $\operatorname{dim}\left(V_{\mathrm{POD}}^{l}\right)=l$ and, in general, $l \ll m N_{h}$.

As to the choice of the integer $l$, different criteria can be adopted. For instance, one can analyze the trend of the spectrum $\Sigma$ or introduce a control on the variance by selecting the first $l$ ordered singular values such that

$$
R_{l}=\frac{\sum_{i=1}^{l} \sigma_{i}^{2}}{\sum_{i=1}^{p} \sigma_{i}^{2}} \geq \epsilon
$$

for a positive user-defined tolerance $\epsilon$ [36].

REMARK 3.1. As an alternative to the procedure above, the POD basis can be derived by applying a spectral decomposition for the covariance matrix $C=\mathcal{V}^{T} \mathcal{V} \in \mathbb{R}^{p \times p}$ assuming $p \ll m N_{h}$. In particular, it holds that $\lambda_{i}=\sigma_{i}^{2}$ and $\phi_{i}=\lambda_{i}^{-1} S \mathbf{c}_{i}$, where $\left\{\lambda_{i}, \mathbf{c}_{i}\right\}$ denotes the generic \{eigenvalue, eigenvector $\}$ pair associated with $C$, for $i=1, \ldots, p$ [36].

REMARK 3.2 (Snaphot choice). The choice of the representative values for the parameter $\alpha$ in (3.2) is a critical issue to make POD effective in practice. In general, it strictly depends on the problem at hand. In particular, the model reduction is effective if the selected snapshots cover the whole parameter space. This aspect is beyond the goal of this work albeit extremely interesting.

Now, the online phase approximates the HiMod solution to problem (2.1) for the value $\alpha^{*}$ of the parameter, with $\alpha^{*} \neq \alpha_{i}$ for $i=1, \ldots, p$, at a lower computational cost with respect to directly solving the HiMod system (2.4) for $\alpha=\alpha^{*}$. For this purpose, we project the system (2.4) onto the POD space $V_{\mathrm{POD}}^{l}$ by computing the POD stiffness matrix and right-hand side,

$$
\begin{aligned}
A_{\mathrm{POD}}\left(\alpha^{*}\right) & =\left(\Phi_{\mathrm{POD}}^{l}\right)^{T} A_{m}\left(\alpha^{*}\right) \Phi_{\mathrm{POD}}^{l} \in \mathbb{R}^{l \times l}, \\
\mathbf{f}_{\mathrm{POD}}\left(\alpha^{*}\right) & =\left(\Phi_{\mathrm{POD}}^{l}\right)^{T} \mathbf{f}_{m}\left(\alpha^{*}\right) \in \mathbb{R}^{l},
\end{aligned}
$$

respectively, where the matrix $\Phi_{\mathrm{POD}}^{l}=\left[\phi_{1}, \ldots, \phi_{l}\right] \in \mathbb{R}^{\left(m N_{h}\right) \times l}$ collects the POD basis vectors column-wise, while $A_{m}\left(\alpha^{*}\right)$ and $\mathbf{f}_{m}\left(\alpha^{*}\right)$ are the HiMod stiffness matrix and right-hand side in (2.4). Then, we solve the POD system of order $l$

$$
A_{\mathrm{POD}}\left(\alpha^{*}\right) \mathbf{u}_{\mathrm{POD}}\left(\alpha^{*}\right)=\mathbf{f}_{\mathrm{POD}}\left(\alpha^{*}\right)
$$




\section{ETNA}

Kent State University and

Johann Radon Institute (RICAM)

with $\mathbf{u}_{\mathrm{POD}}\left(\alpha^{*}\right)=\left[u_{\mathrm{POD}, 1}^{\alpha^{*}}, \ldots, u_{\mathrm{POD}, l}^{\alpha^{*}}\right]^{T} \in \mathbb{R}^{l}$. This allows us to approximate the HiMod solution $\mathbf{u}_{m}\left(\alpha^{*}\right)$ in (2.4) by using the POD basis as

$$
\mathbf{u}_{m}\left(\alpha^{*}\right) \approx \mathbf{u}_{\mathrm{HiPOD}}^{l}\left(\alpha^{*}\right)=\sum_{s=1}^{l} u_{\mathrm{POD}, s}^{\alpha^{*}} \boldsymbol{\phi}_{s} \in \mathbb{R}^{m N_{h}}
$$

after solving a system of order $l$ instead of $m N_{h}$. Finally, thanks to the expansion (2.5), we obtain the HiPOD approximation $u_{\mathrm{HiPOD}}^{l}\left(\alpha^{*}\right)$ to $u_{m}\left(x, \mathbf{y} ; \alpha^{*}\right)$.

The assembly of $A_{m}\left(\alpha^{*}\right)$ and $\mathbf{f}_{m}\left(\boldsymbol{\alpha}^{*}\right)$ in (3.5) constitutes the bottleneck of the basic HiPOD method although this represents a computational burden typical of any projectionbased POD procedure. Nevertheless, the employment of a reduced rather than a full model when building the matrix $S$ leads to a considerable reduction of the computational effort, especially when $m$ is a small value.

3.1.1. Numerical assessment. The basic HiPOD procedure is assessed on the test problems in Section 2.1.

Test case 3. To perform the offline phase, we assume an affine dependence of the problem data in (2.2) on the independent variables $x, y$ so that

$$
\begin{array}{ll}
\mu(\mathbf{x})=\mu_{0}+\mu_{x} x+\mu_{y} y, & \mathbf{b}(\mathbf{x})=\left[b_{0}+b_{x} x, b_{1}+b_{y} y\right]^{T}, \\
\sigma(\mathbf{x})=\sigma_{0}+\sigma_{x} x+\sigma_{y} y, & f(\mathbf{x})=f_{0}+f_{x} x+f_{y} y .
\end{array}
$$

Then, we hierarchically reduce 30 different problems by setting $\mu_{0}=1, \sigma_{x}=\sigma_{y}=0, f_{0}=1$, and by randomly varying the remaining nine parameters as

$$
\begin{aligned}
& \mu_{x} \in \mathcal{P}_{\mu_{x}}=[0,2], \quad \mu_{y} \in \mathcal{P}_{\mu_{y}}=[0,2], \quad \sigma_{0} \in \mathcal{P}_{\sigma_{0}}=[0,3], \\
& b_{0} \in \mathcal{P}_{b_{0}}=[0,3], \quad b_{1} \in \mathcal{P}_{b_{1}}=[0,3], \quad b_{x} \in \mathcal{P}_{b_{x}}=[0,2], \\
& b_{y} \in \mathcal{P}_{b_{y}}=[0,2], \quad f_{x} \in \mathcal{P}_{f_{x}}=[-2,2], \quad f_{y} \in \mathcal{P}_{f_{y}}=[-2,2],
\end{aligned}
$$

so that the parameter in (2.1) coincides with the vector $\boldsymbol{\alpha}=\left[\mu_{x}, \mu_{y}, \sigma_{0}, b_{0}, b_{1}, b_{x}, b_{y}, f_{x}, f_{y}\right]^{T}$ $\in \mathbb{R}^{9}$ varying in $\mathcal{P}=\mathcal{P}_{\mu_{x}} \times \mathcal{P}_{\mu_{y}} \times \mathcal{P}_{\sigma_{0}} \times \mathcal{P}_{b_{0}} \times \mathcal{P}_{b_{1}} \times \mathcal{P}_{b_{x}} \times \mathcal{P}_{b_{y}} \times \mathcal{P}_{f_{x}} \times \mathcal{P}_{f_{y}}$.

The HiMod discretization uses linear finite elements along the mainstream, associated with a uniform partition of $\Omega_{1 D}$ into 60 subintervals, and a modal expansion based on 10 sinusoidal modes. Figure 3.1 (left) displays the spectrum of the matrix $\mathcal{V}$, where the vertical lines identify the dimension $l$ for the POD space adopted in the online phase. The singular values decrease rather slowly until a drop occurs at $l=17(\operatorname{being} \operatorname{rank}(\mathcal{V})=17)$. This can be ascribed to the large number of parameters involved, which limits the redundancy across the snapshots.

During the online phase we approximate the same problem as in Section 2.1.1 for

$$
\boldsymbol{\alpha}^{*}=[0,0,0,3,0,0,0,-2,3]^{T} \in \mathcal{P},
$$

so that the reference HiMod solution is the one in Figure 2.1 (bottom-right). Starting from the spectrum on the left-hand side of Figure 3.1, we pick $l=2,4,7,15,17$. The corresponding value for the ratio $R_{l}$ in (3.4) is given by $0.9352,0.9832,0.9952,0.9999,1$, respectively. Figure 3.2 provides the contour plots of $u_{\mathrm{HiPOD}}^{l}\left(\boldsymbol{\alpha}^{*}\right)$ for $l=2,7,15$. The solutions $u_{\mathrm{HiPOD}}^{2}\left(\boldsymbol{\alpha}^{*}\right)$ and $u_{\mathrm{HiPOD}}^{7}\left(\boldsymbol{\alpha}^{*}\right)$ exhibit a good accuracy if we take into account that they are obtained by solving a system of dimensionality 2 and 7 , respectively, and that we are varying 9 parameters simultaneously. The quality of the HiPOD approximation gradually improves by increasing the dimension of the POD space as confirmed also by the values in Table 3.1, which gathers 

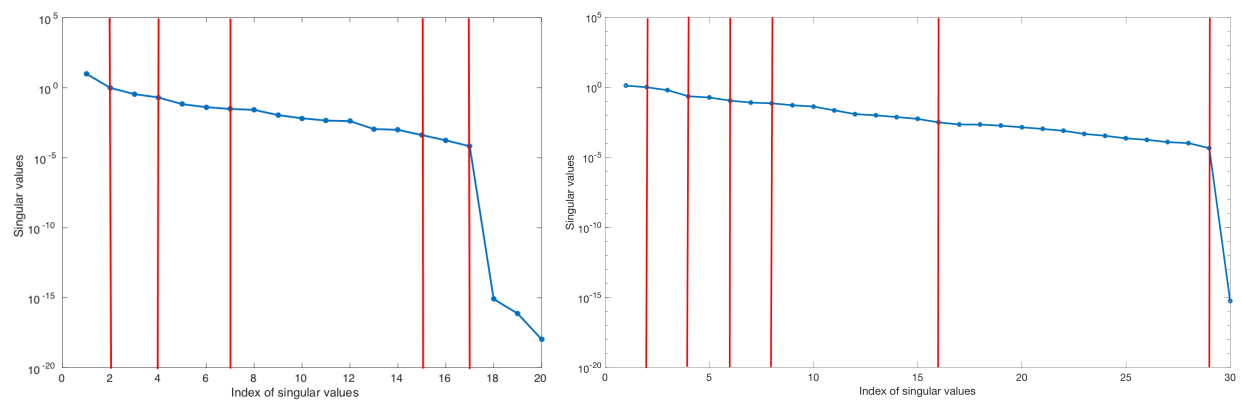

FIG. 3.1. Basic HiPOD reduction: singular values of the matrix $\mathcal{V}$ for the test cases 3 (left) and 4 (right).
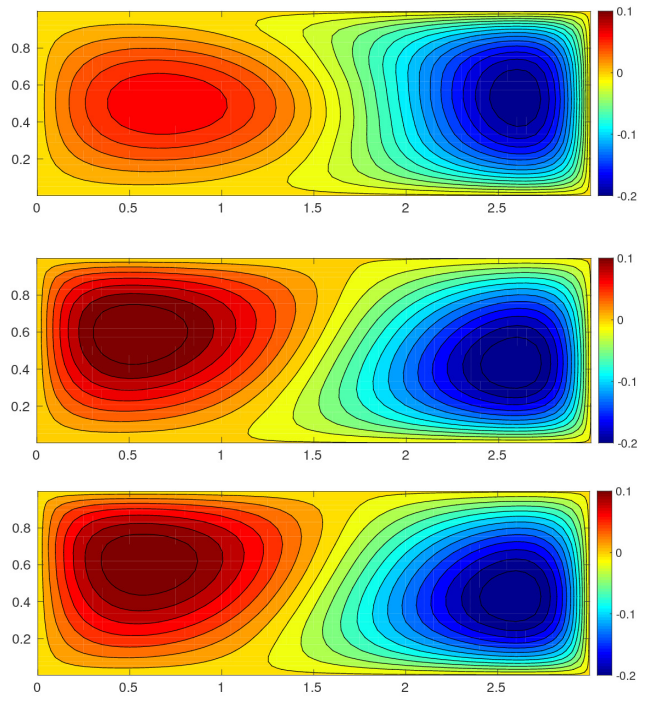

FIG. 3.2. Basic HiPOD reduction (test case 3): HiPOD approximation for $l=2$ (top), $l=7$ (middle), and $l=15$ (bottom).

the $L^{2}(\Omega)$ - and the $H^{1}(\Omega)$-norm of the relative modeling error obtained by replacing the HiMod solution $u_{10}\left(\boldsymbol{\alpha}^{*}\right)$ with the HiPOD approximation $u_{\mathrm{HiPOD}}^{l}\left(\boldsymbol{\alpha}^{*}\right)$ for different values of $l$. The modeling error is quickly reduced by increasing $l$. From a qualitative viewpoint, the HiPOD approximation $u_{\mathrm{HiPOD}}^{15}\left(\boldsymbol{\alpha}^{*}\right)$ is fully comparable with the HiMod approximation in Figure 2.1 (bottom-right) with a reduction of the wall-clock time from 1.44 to 0.04 seconds $^{1}$ (the time associated with the HiPOD approximation refers to the online phase only).

Test case 4. As reference setting, we consider now the test case in Section 2.1.2. We adopt the following dependence on the independent variables for the problem data in (2.1):

$$
\begin{aligned}
\mu(\mathbf{x}) & =\mu_{0}+\mu_{x} x+\mu_{y} y, & \mathbf{b}(\mathbf{x}) & =\left[b_{0}, b_{1} \sin (6 x)\right]^{T}, \\
\sigma(\mathbf{x}) & =\sigma_{0}+\sigma_{x} x+\sigma_{y} y, & f(\mathbf{x}) & =f_{1} \chi_{C_{1}}(\mathbf{x})+f_{2} \chi_{C_{2}}(\mathbf{x}) .
\end{aligned}
$$

\footnotetext{
${ }^{1}$ The computations have been run on a MacBookPro15,3 Intel Core i9 2.40GHz 32 GB desktop computer.
} 
TABLE 3.1

Basic HiPOD reduction (test case 3): relative modeling error for different HiPOD approximations.

\begin{tabular}{c|c|c|c|c|c} 
& $l=2$ & $l=4$ & $l=7$ & $l=15$ & $l=17$ \\
\hline$L^{2}(\Omega)$-norm & $3.23 \mathrm{e}-01$ & $5.98 \mathrm{e}-02$ & $3.51 \mathrm{e}-02$ & $2.70 \mathrm{e}-03$ & $1.71 \mathrm{e}-03$ \\
$H^{1}(\Omega)$-norm & $4.50 \mathrm{e}-01$ & $1.23 \mathrm{e}-01$ & $6.21 \mathrm{e}-02$ & $7.61 \mathrm{e}-03$ & $4.81 \mathrm{e}-03$
\end{tabular}

TABLE 3.2

Basic HiPOD reduction (test case 4): relative modeling error for different HiPOD approximations.

\begin{tabular}{c|c|c|c|c|c|c} 
& $l=2$ & $l=4$ & $l=6$ & $l=8$ & $l=16$ & $l=29$ \\
\hline$L^{2}(\Omega)$-norm & $2.41 \mathrm{e}-01$ & $2.12 \mathrm{e}-01$ & $9.83 \mathrm{e}-02$ & $3.42 \mathrm{e}-02$ & $3.94 \mathrm{e}-03$ & $1.23 \mathrm{e}-03$ \\
$H^{1}(\Omega)$-norm & $3.23 \mathrm{e}-01$ & $1.87 \mathrm{e}-01$ & $1.15 \mathrm{e}-01$ & $4.93 \mathrm{e}-02$ & $9.31 \mathrm{e}-03$ & $2.33 \mathrm{e}-03$
\end{tabular}

During the offline phase, we compute the HiMod approximation for 30 different ADR problems by setting $\mu_{x}=\mu_{y}=\sigma_{x}=\sigma_{y}=0$ and by randomly varying

$$
\begin{aligned}
& \mu_{0} \in \mathcal{P}_{\mu_{0}}=[0.1,10], \quad b_{0} \in \mathcal{P}_{b_{0}}=[2,20], \quad b_{1} \in \mathcal{P}_{b_{1}}=[1,3], \\
& \sigma_{0} \in \mathcal{P}_{\sigma_{0}}=[0,3], \quad f_{1} \in \mathcal{P}_{f_{1}}=[5,25], \quad f_{2} \in \mathcal{P}_{f_{2}}=[5,25] \text {, }
\end{aligned}
$$

so that the parameter in (2.1) is provided by the vector $\boldsymbol{\alpha}=\left[\mu_{0}, b_{0}, b_{1}, \sigma_{0}, f_{1}, f_{2}\right]^{T}$ taking values in the set $\mathcal{P}=\mathcal{P}_{\mu_{0}} \times \mathcal{P}_{b_{0}} \times \mathcal{P}_{b_{1}} \times \mathcal{P}_{\sigma_{0}} \times \mathcal{P}_{f_{1}} \times \mathcal{P}_{f_{2}}$. The HiMod discretization employs linear finite elements on a uniform partition of $\Omega_{1 D}$ into 120 subintervals combined with 20 sinusoidal modes to discretize the transverse dynamics. Figure 3.1 (right) illustrates the trend of the spectrum for the corresponding matrix $\mathcal{V}$. This is characterized by a very slow decay without any significant drop before the 29th singular value (being $\operatorname{rank}(\mathcal{V})=29$ ).

The online phase is employed to approximate the solution for the problem in Section 2.1.2. This is equivalent to the parameter setting

$$
\boldsymbol{\alpha}^{*}=[0.24,5,1,0.1,10,10]^{T} \in \mathcal{P} .
$$

Figure 3.3 (top-bottom) displays the basic HiPOD approximations $u_{\mathrm{HiPOD}}^{2}\left(\boldsymbol{\alpha}^{*}\right), u_{\mathrm{HiPOD}}^{6}\left(\boldsymbol{\alpha}^{*}\right)$, $u_{\mathrm{HiPOD}}^{16}\left(\boldsymbol{\alpha}^{*}\right)$. As expected, the ratio $R_{l}$ becomes closer to 1 when $l$ increases, being $R_{2}=0.6163, R_{6}=0.9158$, and $R_{16}=0.9971$. Six POD modes suffice to recognize already the general trend of the HiMod solution, whereas the HiPOD approximation $u_{\mathrm{HiPOD}}^{16}\left(\boldsymbol{\alpha}^{*}\right)$, which is obtained by solving a system of order 16 , is fully comparable with the HiMod approximation $u_{5}\left(\boldsymbol{\alpha}^{*}\right)$ in Figure 2.2 (bottom), a solution of a system of dimension 600 . This leads to a significant saving in terms of the computational effort, the wall-clock time reducing from 14.53 seconds for the HiMod approximation to 0.20 seconds when resorting to the basic HiPOD approach.

Finally, Table 3.2 provides some quantitative information about the accuracy of the basic HiPOD approximation by collecting the $L^{2}(\Omega)$ - and the $H^{1}(\Omega)$-norm of the relative modeling error with respect to the HiMod approximation $u_{20}\left(\boldsymbol{\alpha}^{*}\right)$. The error reduction is slightly slower compared with the values in Table 3.1, the trend of the solution being in such a case less trivial.

3.2. The directional HiPOD approach. The directional HiPOD method combines HiMod reduction with POD by a deeper exploitation of the separation of variables underlying a HiMod discretization. In particular, the SVD is employed to erase the redundancy along the main stream and the transverse direction separately. Then, the online phase is carried out by interpolating instead of projecting. This relieves us from assembling the HiMod stiffness matrix and the right-hand side associated with the online parameter as expected by (3.5). 


\section{ETNA}

Kent State University and Johann Radon Institute (RICAM)
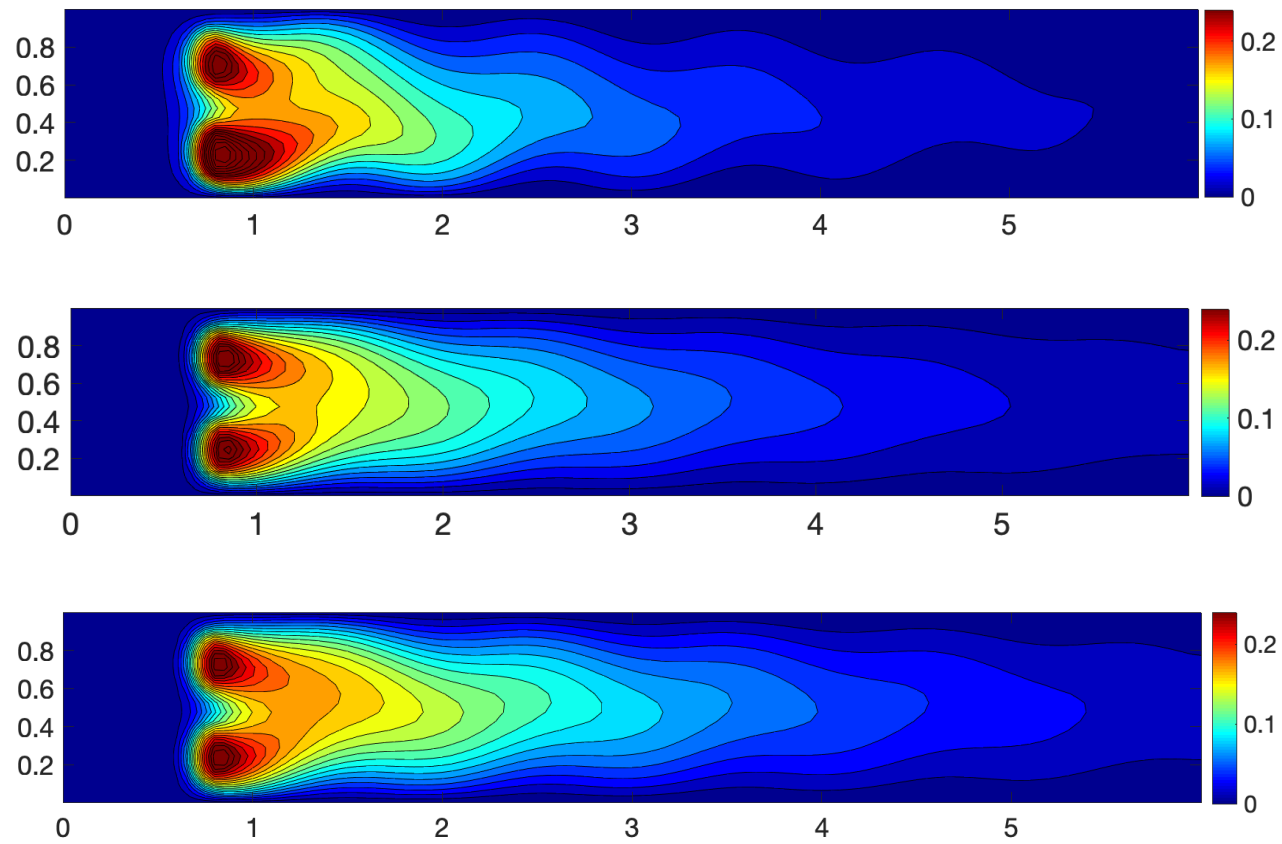

FIG. 3.3. Basic HiPOD reduction (test case 4): HiPOD approximation for $l=2$ (top), $l=6$ (center), and $l=16($ bottom).

The offline phase starts by collecting the information to build the response matrix. To this aim, we compute the HiMod discretization for problem (2.1) for $p$ different values $\alpha_{i}$ of the parameter $\alpha$, with $i=1, \ldots, p$. The corresponding modal coefficients $\left\{\widetilde{u}_{k, j}^{\alpha_{i}}\right\}_{k=1, j=1}^{m, N_{h}}$ are re-ordered by mode into the $m$ vectors

$$
\mathbf{U}^{k}\left(\alpha_{i}\right)=\left[\widetilde{u}_{k, 1}^{\alpha_{i}}, \widetilde{u}_{k, 2}^{\alpha_{i}}, \ldots, \widetilde{u}_{k, N_{h}}^{\alpha_{i}}\right]^{T} \in \mathbb{R}^{N_{h}}, \quad k=1, \ldots, m
$$

instead of in a unique vector as in (3.1). Then, we employ the vectors $\mathbf{U}^{k}\left(\alpha_{i}\right)$ to assemble the response matrix

$$
\begin{aligned}
& U=\left[\mathbf{U}^{1}\left(\alpha_{1}\right) \cdots \mathbf{U}^{m}\left(\alpha_{1}\right)\left|\mathbf{U}^{1}\left(\alpha_{2}\right) \ldots \mathbf{U}^{m}\left(\alpha_{2}\right)\right| \cdots \ldots \mid \mathbf{U}^{1}\left(\alpha_{p}\right) \cdots \mathbf{U}^{m}\left(\alpha_{p}\right)\right] \\
& =\left[\begin{array}{ccc|ccc|cc|ccc}
\widetilde{u}_{1,1}^{\alpha_{1}} & \ldots & \widetilde{u}_{m, 1}^{\alpha_{1}} & \widetilde{u}_{1,1}^{\alpha_{2}} & \ldots & \widetilde{u}_{m, 1}^{\alpha_{2}} & \ldots & \ldots & \widetilde{u}_{1,1}^{\alpha_{p}} & \ldots & \widetilde{u}_{m, 1}^{\alpha_{p}} \\
\widetilde{u}_{1,2}^{\alpha_{1}} & \ldots & \widetilde{u}_{m, 2}^{\alpha_{1}} & \widetilde{u}_{1,2}^{\alpha_{2}} & \ldots & \widetilde{u}_{m, 2}^{\alpha_{2}} & \ldots & \ldots & \widetilde{u}_{1,2}^{\alpha_{p}} & \ldots & \widetilde{u}_{m, 2}^{\alpha_{p}} \\
\vdots & \vdots & \vdots & \vdots & \vdots & \vdots & \vdots & \vdots & \vdots & \vdots & \vdots \\
\widetilde{u}_{1, N_{h}}^{\alpha_{1}} & \cdots & \widetilde{u}_{m, N_{h}}^{\alpha_{1}} & \widetilde{u}_{1, N_{h}}^{\alpha_{2}} & \ldots & \widetilde{u}_{m, N_{h}}^{\alpha_{2}} & \cdots & \ldots & \widetilde{u}_{1, N_{h}}^{\alpha_{p}} & \ldots & \widetilde{u}_{m, N_{h}}^{\alpha_{p}}
\end{array}\right] .
\end{aligned}
$$

The matrix $U \in \mathbb{R}^{N_{h} \times(m p)}$ exhibits a block-wise structure associated with the parameters $\alpha_{i}$ such that, for each block, the columns run over the modes while the rows run over the finite element nodes. Now, we apply the SVD to the matrix $U$, thus yielding

$$
U=\Xi \Lambda K^{T}
$$

with $\Xi \in \mathbb{R}^{N_{h} \times N_{h}}$ and $K \in \mathbb{R}^{(m p) \times(m p)}$ being unitary matrices and $\Lambda \in \mathbb{R}^{N_{h} \times(m p)}$ a pseudo-diagonal matrix. The left singular vectors $\left\{\boldsymbol{\xi}_{j}\right\}_{j=1}^{N_{h}}$ of $U$ constitute an orthogonal basis 
for $\mathbb{R}^{N_{h}}$ so that each column of $U$ can be expanded as

$$
\mathbf{U}^{k}\left(\alpha_{i}\right)=\sum_{j=1}^{N_{h}} T_{j}^{k}\left(\alpha_{i}\right) \boldsymbol{\xi}_{j}, \quad k=1, \ldots, m, i=1, \ldots, p .
$$

In general, we can pick the first, say $L$ with $L \leq N_{h}$, most meaningful singular vectors of $U$ to identify the POD space $V_{\mathrm{POD}, 1}^{L}=\operatorname{span}\left\{\boldsymbol{\xi}_{1}, \ldots, \boldsymbol{\xi}_{L}\right\}$ associated with this first phase of the directional HiPOD procedure with $\operatorname{dim}\left(V_{\mathrm{POD}, 1}^{L}\right)=L$. Thus, the vectors $\mathbf{U}^{k}\left(\alpha_{i}\right)$ can be approximated as

$$
\mathbf{U}^{k}\left(\alpha_{i}\right) \cong \sum_{j=1}^{L} T_{j}^{k}\left(\alpha_{i}\right) \boldsymbol{\xi}_{j}, \quad k=1, \ldots, m, i=1, \ldots, p,
$$

where equality holds when $L=N_{h}$ (see (3.8)). Now, we re-organize the coefficients $\left\{T_{j}^{k}\left(\alpha_{i}\right)\right\}$ by parameter into the $p$ vectors $\mathbf{T}_{j}\left(\alpha_{i}\right)=\left[T_{j}^{1}\left(\alpha_{i}\right), \ldots, T_{j}^{m}\left(\alpha_{i}\right)\right]^{T} \in \mathbb{R}^{m}$, with $i=1, \ldots, p$, and we define the matrix

$$
S_{j}=\left[\mathbf{T}_{j}\left(\alpha_{1}\right), \ldots, \mathbf{T}_{j}\left(\alpha_{p}\right)\right]=\left[\begin{array}{ccc}
T_{j}^{1}\left(\alpha_{1}\right) & \ldots & T_{j}^{1}\left(\alpha_{p}\right) \\
\vdots & & \vdots \\
T_{j}^{m}\left(\alpha_{1}\right) & \ldots & T_{j}^{m}\left(\alpha_{p}\right)
\end{array}\right] \in \mathbb{R}^{m \times p}
$$

with $j=1, \ldots, L$. Then, we apply the SVD to each matrix $S_{j}$ to obtain the $L$ factorizations

$$
S_{j}=R_{j} D_{j} P_{j}^{T}
$$

with $R_{j} \in \mathbb{R}^{m \times m}$ and $P_{j} \in \mathbb{R}^{p \times p}$ being unitary matrices and $D_{j} \in \mathbb{R}^{m \times p}$ the pseudodiagonal matrix collecting the singular values of $S_{j}$. Thus, the columns $\mathbf{T}_{j}\left(\alpha_{i}\right)$ of $S_{j}$ can be represented in terms of the POD orthogonal basis $\left\{\mathbf{r}_{j}^{k}\right\}_{k=1}^{\mu_{j}}$, with $\mu_{j} \leq m$, constituted by the most significant $\mu_{j}$ left singular vectors of $S_{j}$, as

$$
\mathbf{T}_{j}\left(\alpha_{i}\right) \cong \sum_{k=1}^{\mu_{j}} Q_{j}^{k}\left(\alpha_{i}\right) \mathbf{r}_{j}^{k}, \quad j=1, \ldots, L, i=1, \ldots, p .
$$

With each $j$, we associate the POD space $V_{\mathrm{POD}, 2, j}^{\mu_{j}}=\operatorname{span}\left\{\mathbf{r}_{j}^{1}, \ldots, \mathbf{r}_{j}^{\mu_{j}}\right\}$ of dimension $\operatorname{dim}\left(V_{\mathrm{POD}, 2, j}^{\mu_{j}}\right)=\mu_{j}$. Thus, the directional HiPOD procedure yields $(L+1)$ POD bases, which, during the online phase, are employed to predict the HiMod approximation to the problem (2.1) for a new value $\alpha^{*}$ of the parameter with $\alpha^{*} \neq \alpha_{i}$, for $i=1, \ldots, p$.

For this purpose, first we compute an approximation for the coefficients $Q_{j}^{k}\left(\alpha^{*}\right)$ in (3.11), for $j=1, \ldots, L$ and $k=1, \ldots, \mu_{j}$, via a suitable interpolation of the (known) values $Q_{j}^{k}\left(\alpha_{i}\right)$, for $i=1, \ldots, p$. Successively, we go through the directional procedure backward until we obtain an approximation for the vector $\mathbf{U}^{k}\left(\alpha^{*}\right)$ in (3.6). In particular, thanks to (3.11), we compute the $L$ vectors

$$
\mathbf{T}_{j}\left(\alpha^{*}\right)=\left[T_{j}^{1}\left(\alpha^{*}\right), \ldots, T_{j}^{m}\left(\alpha^{*}\right)\right]^{T}=\sum_{k=1}^{\mu_{j}} Q_{j}^{k}\left(\alpha^{*}\right) \mathbf{r}_{j}^{k}, \quad j=1, \ldots, L
$$

in $\mathbb{R}^{m}$, and then, according to (3.9), we assemble the $m$ vectors $\mathbf{U}_{\mathrm{HiPOD}}^{k}\left(\alpha^{*}\right) \in \mathbb{R}^{N_{h}}$ as

$$
\mathbf{U}_{\mathrm{HiPOD}}^{k}\left(\alpha^{*}\right)=\left[u_{\mathrm{POD}, k, 1}^{\alpha^{*}}, \ldots, u_{\mathrm{POD}, k, N_{h}}^{\alpha^{*}}\right]^{T}=\sum_{j=1}^{L} T_{j}^{k}\left(\alpha^{*}\right) \boldsymbol{\xi}_{j}, \quad k=1, \ldots, m .
$$




\section{ETNA}

Kent State University and

Johann Radon Institute (RICAM)

HIERARCHICAL MODEL REDUCTION DRIVEN BY POD

TABLE 3.3

Directional HiPOD reduction (test case 5): prediction for the POD modes.

\begin{tabular}{c|c|c|c|c|c} 
& $\varepsilon=0.6$ & $\varepsilon=0.9$ & $\varepsilon=0.99$ & $\varepsilon=0.999$ & $\varepsilon=0.9999$ \\
\hline$L$ & 1 & 3 & 5 & 7 & 10 \\
$\max _{j} \mu_{j}$ & 4 & 6 & 8 & 9 & 10 \\
$\operatorname{median}_{j}$ & 2 & 4 & 6 & 8 & 8
\end{tabular}

Finally, the vectors $\mathbf{U}_{\mathrm{HiPOD}}^{k}\left(\alpha^{*}\right)$ allow us to approximate the HiMod solution $u_{m}\left(\alpha^{*}\right)$ as

$$
u_{m}\left(\alpha^{*}\right) \approx u_{\mathrm{HiPOD}}^{L, M_{L}}\left(\alpha^{*}\right)=\sum_{k=1}^{m}\left[\sum_{j=1}^{N_{h}} u_{\mathrm{POD}, k, j}^{\alpha^{*}} \vartheta_{j}(x)\right] \varphi_{k}\left(\psi_{x}(\mathbf{y})\right),
$$

with $M_{L}=\left\{\mu_{j}\right\}_{j=1}^{L}$, and where the values $u_{\mathrm{POD}, k, j}^{\alpha^{*}}$ provide an approximation of the actual coefficient $\widetilde{u}_{k, j}^{\alpha^{*}}$ in (3.6) with $\alpha_{i}=\alpha^{*}$.

REMARK 3.3 (Choice of the interpolation). Different interpolation procedures can be adopted to compute the coefficients $Q_{j}^{k}\left(\alpha^{*}\right)$. Following [37], we adopt a standard linear interpolation, a piecewise cubic Hermite interpolant, and an interpolating radial basis function. In the next section, we numerically investigate the performances of these three approaches.

3.2.1. Numerical assessment. We numerically assess the reliability of the directional HiPOD procedure. First, we consider the case where $\alpha$ coincides with a single scalar quantity, and then we generalize the approach to the vector case so that $\alpha$ will collect several parameters.

Test case 5. We adopt the solution for test case 1 as the setting to be approximated during the online phase. The viscosity coefficient $\mu$, which is here assumed constant, represents the parameter driving the offline phase so that $\alpha=\mu$. In particular, we hierarchically reduce problem (2.1)-(2.2) for 20 different values of $\mu$ with $\mu=\mu_{i}$ uniformly sampled in the interval $\mathcal{P}_{\mu}=[0.15,3]$ and $\mu_{i} \neq 1$, for $i=1, \ldots, 20$, while preserving the same values as in (2.6) for the other problem data. The HiMod discretization is the same as for test case 3 so that we employ linear finite elements associated with a uniform partition of $\Omega_{1 D}$ into 60 subintervals to discretize the main stream and 10 sinusoidal modes to describe the transverse dynamics.

Concerning the choice of $L$ in (3.9) and of $\mu_{i}$ in (3.11), we resort to a control analogous to the one in (3.4). In more detail, for two fixed tolerances $\varepsilon_{1}$ and $\varepsilon_{2}$ with $0 \leq \varepsilon_{1}, \varepsilon_{2} \leq 1$, we preserve the first $L$ left singular vectors $\boldsymbol{\xi}_{j}$ of $U$ and the first $\mu_{j}$ left singular vectors $\mathbf{r}_{j}^{k}$ of $S_{j}$ such that

$$
R_{\mathrm{POD}, 1}^{L}=\frac{\sum_{j=1}^{L} \lambda_{j}^{2}}{\sum_{j=1}^{N_{h}} \lambda_{j}^{2}} \geq \varepsilon_{1} \quad \text { and } \quad R_{\mathrm{POD}, 2}^{\mu_{j}}=\frac{\sum_{k=1}^{\mu_{j}} d_{j, k}^{2}}{\sum_{k=1}^{m} d_{j, k}^{2}} \geq \varepsilon_{2},
$$

respectively, with $\lambda_{j}$ the singular value of $U$ associated with $\boldsymbol{\xi}_{j}$ and $j=1, \ldots, N_{h}$ and with $d_{j, k}$ the singular value of $S_{j}$ corresponding to the $k$ th singular vector $\mathbf{r}_{j}^{k}$ and $k=1, \ldots, m$. As a first test, we choose $\varepsilon_{1}=\varepsilon_{2}=\varepsilon$. Table 3.3 collects the predictions for $L$ for the maximum value and for the median of the values $\mu_{j}$ for different choices of $\varepsilon$. As expected, the number of Hi-POD modes retained at both stages increases when $\varepsilon$ approaches 1 . Moreover, a slightly higher sensitivity of $L$ with respect to the selected tolerance is detected when compared with the maximum value and the median of the $\mu_{j}$.

The online phase is performed by setting $\alpha^{*}=\mu^{*}=1 \in \mathcal{P}_{\mu}$ and by using a radial basis function (RBF) interpolation [38]. In Figure 3.4, we compare the HiPOD approximations associated with three of the selected tolerances. It is noticed that at the first level of the procedure at least three POD modes have to be adopted to have an approximation sufficiently 

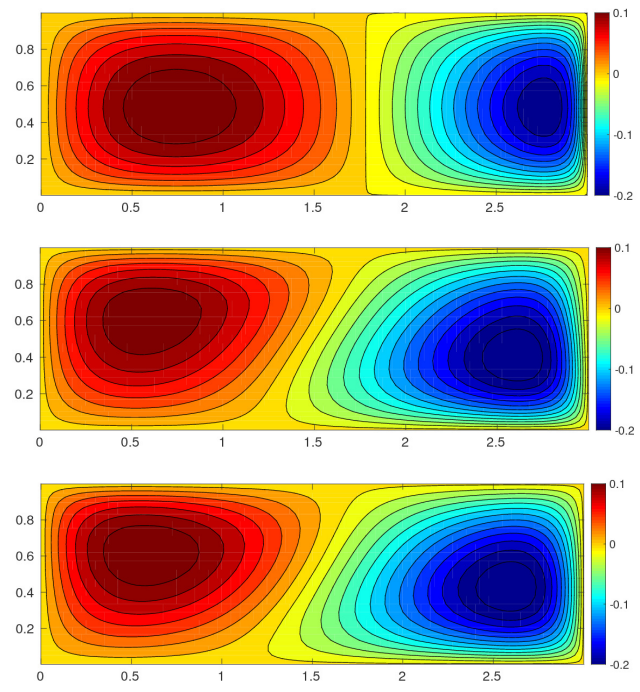

FIG. 3.4. Directional HiPOD reduction (test case 5): HiPOD approximation for $\varepsilon=0.6$ (top), $\varepsilon=0.9$ (middle), and $\varepsilon=0.99$ (bottom).

TABLE 3.4

Directional HiPOD reduction (test case 5): relative modeling error for different HiPOD approximations.

\begin{tabular}{c|c|c|c|c|c} 
& $\varepsilon=0.6$ & $\varepsilon=0.9$ & $\varepsilon=0.99$ & $\varepsilon=0.999$ & $\varepsilon=0.9999$ \\
\hline$L^{2}(\Omega)$-norm & $4.66 \mathrm{e}-01$ & $4.01 \mathrm{e}-02$ & $3.11 \mathrm{e}-03$ & $8.17 \mathrm{e}-04$ & $1.37 \mathrm{e}-04$ \\
$H^{1}(\Omega)$-norm & $4.62 \mathrm{e}-01$ & $9.43 \mathrm{e}-02$ & $1.05 \mathrm{e}-02$ & $3.11 \mathrm{e}-03$ & $5.71 \mathrm{e}-04$
\end{tabular}

reliable, which is equivalent to picking $\varepsilon \geq 0.9$. On average, the wall-clock time required by the directional HiPOD procedure is 0.08 seconds, which is significantly lower when compared with the time associated with the HiMod reduction in Section 2.1.1 (1.44 seconds for $m=2)$.

In Table 3.4, we analyze the convergence of the directional HiPOD approach by computing the $L^{2}(\Omega)$ - and the $H^{1}(\Omega)$-norm of the error that we have when replacing the HiMod solution $u_{10}\left(\alpha^{*}\right)$ with the HiPOD approximation. The accuracy ensured by the HiPOD approximation is remarkable if we consider that the values in the table refer to a relative error.

Finally, we run the directional HiPOD procedure by distinguishing the tolerances in (3.13) in order to identify a possible criterion for the choice of $\varepsilon_{1}$ and $\varepsilon_{2}$. For this goal, we repeat the same error analysis as in Table 3.4, varying both $\varepsilon_{1}$ and $\varepsilon_{2}$ in the set of values $\{0.6,0.9,0.99,0.999,0.9999\}$. Table 3.5 collects the results of this investigation. It turns out that the values of $\varepsilon_{1}$ and $\varepsilon_{2}$ have to be, in general, sufficiently close to 1 to have a monotonically decreasing trend of the error when we fix a tolerance and vary the other one. For this particular test case, a possible strategy to ensure this monotonicity can be to select $\varepsilon_{1}$ very close to $1\left(\varepsilon_{1}=0.9999\right)$ and make $\varepsilon_{2}$ varying, or, as an alternative, we can fix $\varepsilon_{2}$ to 0.99 , 0.999 , or 0.9999 and gradually reduce the value for $\varepsilon_{1}$. This behaviour is shared by both norms.

Test case 6. The benchmark configuration is now provided by test case 2 , where the HiPOD parameter $\alpha$ coincides with the reactive coefficient $\sigma$ that we assume constant.

The offline phase involves the hierarchically reduction of problem (2.1)-(2.2) for 30 different values of the reaction, uniformly sampled in the range $\mathcal{P}_{\sigma}=[0.02,0.4]$, while all the other problem data in (2.7) are preserved. The HiMod discretization adopted during this 


\section{ETNA}

Kent State University and

Johann Radon Institute (RICAM)

HIERARCHICAL MODEL REDUCTION DRIVEN BY POD

TABLE 3.5

Directional HiPOD reduction (test case 5): sensitivity to the selected tolerances.

\begin{tabular}{l|c|c|c|c|c|c} 
& Norm & $\varepsilon_{2}=0.6$ & $\varepsilon_{2}=0.9$ & $\varepsilon_{2}=0.99$ & $\varepsilon_{2}=0.999$ & $\varepsilon_{2}=0.9999$ \\
\hline$\varepsilon_{1}=0.6$ & $L^{2}(\Omega)$ & $2.58 \mathrm{e}-01$ & $2.58 \mathrm{e}-01$ & $2.57 \mathrm{e}-01$ & $2.57 \mathrm{e}-01$ & $2.57 \mathrm{e}-01$ \\
& $H^{1}(\Omega)$ & $4.61 \mathrm{e}-01$ & $4.61 \mathrm{e}-01$ & $4.59 \mathrm{e}-01$ & $4.59 \mathrm{e}-01$ & $4.59 \mathrm{e}-01$ \\
\hline$\varepsilon_{1}=0.9$ & $L^{2}(\Omega)$ & $5.43 \mathrm{e}-02$ & $5.43 \mathrm{e}-02$ & $2.01 \mathrm{e}-02$ & $2.01 \mathrm{e}-02$ & $2.01 \mathrm{e}-02$ \\
& $H^{1}(\Omega)$ & $1.50 \mathrm{e}-01$ & $1.50 \mathrm{e}-01$ & $5.91 \mathrm{e}-02$ & $5.88 \mathrm{e}-02$ & $5.88 \mathrm{e}-02$ \\
\hline$\varepsilon_{1}=0.99$ & $L^{2}(\Omega)$ & $3.73 \mathrm{e}-02$ & $3.47 \mathrm{e}-02$ & $5.80 \mathrm{e}-03$ & $5.80 \mathrm{e}-03$ & $5.80 \mathrm{e}-03$ \\
& $H^{1}(\Omega)$ & $8.90 \mathrm{e}-02$ & $7.57 \mathrm{e}-02$ & $2.83 \mathrm{e}-02$ & $2.83 \mathrm{e}-02$ & $2.83 \mathrm{e}-02$ \\
\hline$\varepsilon_{1}=0.999$ & $L^{2}(\Omega)$ & $3.72 \mathrm{e}-02$ & $3.46 \mathrm{e}-02$ & $1.34 \mathrm{e}-03$ & $6.03 \mathrm{e}-04$ & $6.03 \mathrm{e}-04$ \\
& $H^{1}(\Omega)$ & $8.85 \mathrm{e}-02$ & $7.51 \mathrm{e}-02$ & $4.01 \mathrm{e}-03$ & $3.10 \mathrm{e}-03$ & $2.91 \mathrm{e}-03$ \\
\hline$\varepsilon_{1}=0.9999$ & $L^{2}(\Omega)$ & $3.72 \mathrm{e}-02$ & $3.46 \mathrm{e}-02$ & $1.20 \mathrm{e}-03$ & $5.57 \mathrm{e}-04$ & $8.03 \mathrm{e}-05$ \\
& $H^{1}(\Omega)$ & $8.84 \mathrm{e}-02$ & $7.50 \mathrm{e}-02$ & $2.81 \mathrm{e}-03$ & $1.21 \mathrm{e}-03$ & $3.95 \mathrm{e}-04$
\end{tabular}

TABLE 3.6

Directional HiPOD reduction (test case 6): prediction for the POD modes.

\begin{tabular}{c|c|c|c|c|c} 
& $\varepsilon=0.6$ & $\varepsilon=0.9$ & $\varepsilon=0.99$ & $\varepsilon=0.999$ & $\varepsilon=0.9999$ \\
\hline$L$ & 2 & 3 & 4 & 12 & 17 \\
$\max _{j} \mu_{j}$ & 5 & 8 & 9 & 10 & 10 \\
$\operatorname{median}_{j}$ & 4 & 5 & 6 & 7 & 7
\end{tabular}

stage uses linear finite elements along $\Omega_{1 D}$ in correspondence with a uniform partition of the supporting fiber into 120 subintervals and 20 sinusoidal modes in the transverse direction, analogously to what is done in test case 4 . We set $\alpha^{*}=\sigma^{*}=0.1 \in \mathcal{P}_{\sigma}$ in the online phase to recover the setting of interest. The spectrum truncation in (3.13) is first driven by a unique tolerance by selecting $\varepsilon_{1}=\varepsilon_{2}=\varepsilon$.

The first row in Table 3.6 provides the number $L$ of POD modes selected at the first level of the HiPOD procedure for five different choices of $\varepsilon$. The values in the table highlight the presence of a strong redundancy. Indeed, $L$ is considerably lower with respect to $N_{h}(=120)$, even when $\varepsilon$ is very close to 1 . For instance, it suffices that the POD space $V_{\mathrm{POD}, 1}^{L}$ has a dimension equal to 12 to correctly describe the dynamics along the main stream. This is shown in Figure 3.5, which gathers the contour plots of the HiPOD approximation associated with the first four values selected for $\varepsilon$. Information about the values predicted for the dimensions $\mu_{j}$ in (3.11) are also furnished by Table 3.6.

The configuration explored in this test case is more complex with respect to the one in test case 5 . This is confirmed by the larger number of POD modes ( $L=12$ versus $L=3$ ) employed at the first level to ensure a reliable HiPOD solution. Despite that, also for this test case we have a computational gain with respect to the HiMod discretization in Section 2.1.2 (case $m=5$ ). Indeed, the wall-clock time characterizing the directional HiPOD procedure is 0.33 seconds to be compared with 14.53 seconds for the HiMod reduction.

The accuracy of the directional HiPOD approximation is quantified in Table 3.7 in terms

TABLE 3.7

Directional HiPOD reduction (test case 6): relative modeling error for different HiPOD approximations.

\begin{tabular}{c|c|c|c|c|c} 
& $\varepsilon=0.6$ & $\varepsilon=0.9$ & $\varepsilon=0.99$ & $\varepsilon=0.999$ & $\varepsilon=0.9999$ \\
\hline$L^{2}(\Omega)$-norm & $1.93 \mathrm{e}-01$ & $7.38 \mathrm{e}-02$ & $1.16 \mathrm{e}-02$ & $4.82 \mathrm{e}-04$ & $5.54 \mathrm{e}-05$ \\
$H^{1}(\Omega)$-norm & $4.34 \mathrm{e}-01$ & $1.52 \mathrm{e}-01$ & $3.08 \mathrm{e}-02$ & $3.11 \mathrm{e}-03$ & $3.84 \mathrm{e}-04$
\end{tabular}



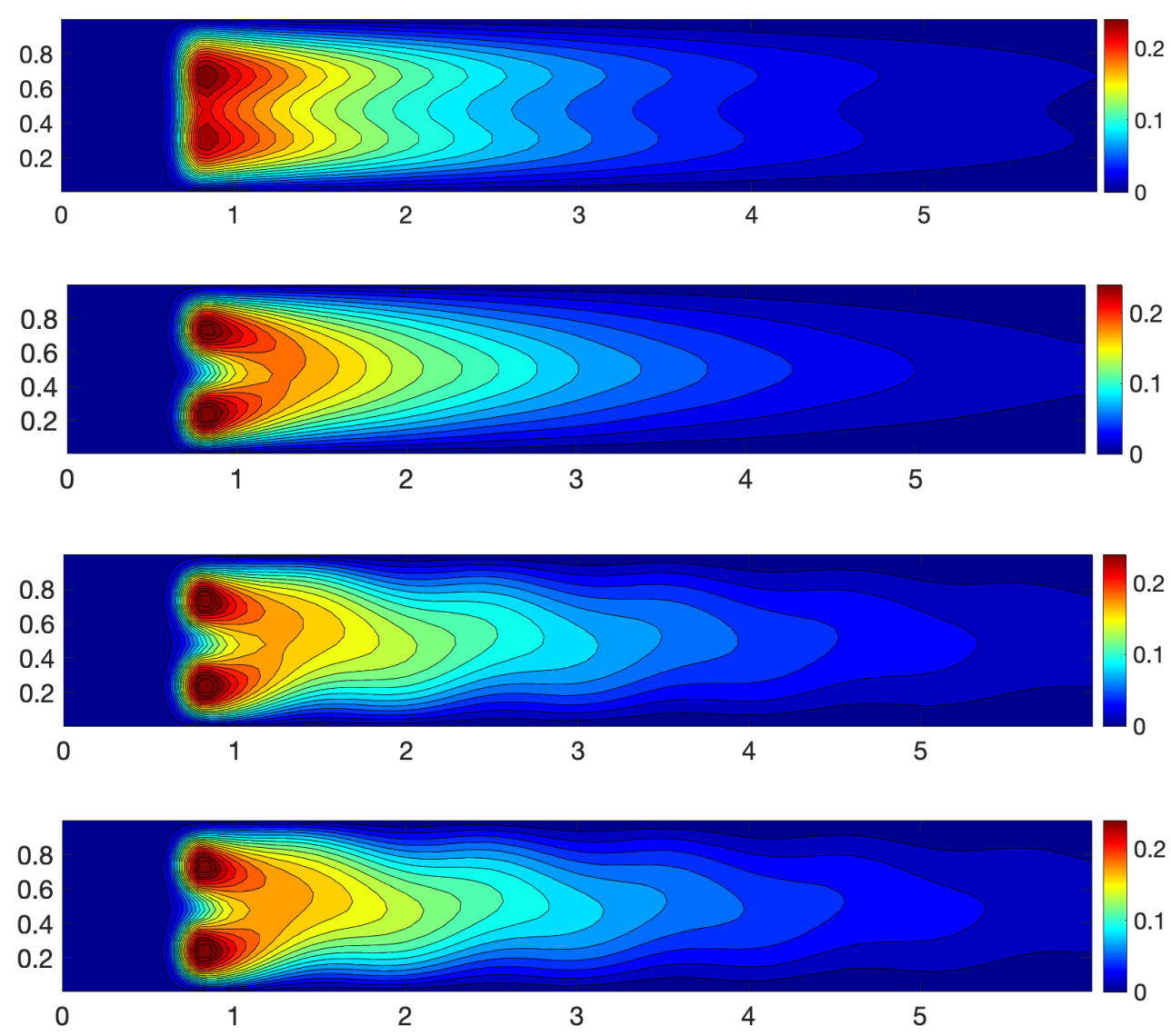

FIG. 3.5. Directional HiPOD reduction (test case 6): HiPOD approximation for $\varepsilon=0.6,0.9,0.99,0.999$ (top-bottom).

of the $L^{2}(\Omega)$ - and of the $H^{1}(\Omega)$-norm of the POD relative error with respect to the HiMod solution $u_{20}\left(\alpha^{*}\right)$. The values in the table confirm the effectiveness of the directional HiPOD procedure.

Also for this test configuration, we explore the accuracy of the directional HiPOD approximation when we select different values for $\varepsilon_{1}$ and $\varepsilon_{2}$. The analysis in Table 3.7 is replicated by assigning the values $0.6,0.9,0.99,0.999,0.9999$ to both the tolerances. Table 3.8 provides the relative modeling error with respect to the reference HiMod solution in terms of the $L^{2}(\Omega)$ and of the $H^{1}(\Omega)$-norms. Conclusions similar to the ones for Table 3.5 can be drawn also for this test case. To ensure a monotonic trend for the error norm, it is fundamental to choose $\varepsilon_{1}$ very close to 1 ( $\varepsilon_{1}=0.999$ or $\left.\varepsilon_{1}=0.9999\right)$ and gradually reduce $\varepsilon_{2}$, or, as an alternative, to set $\varepsilon_{2}$ to $0.99,0.999$, or 0.999 while diminishing $\varepsilon_{1}$.

Finally, we use this test case to investigate the sensitivity of the directional HiPOD reduction procedure to the interpolant used in (3.12) to compute the coefficients $Q_{j}^{k}\left(\alpha^{*}\right)$. For this purpose, we come back to the configuration analyzed in Table 3.7 (i.e., we pick $\varepsilon_{1}=\varepsilon_{2}=\varepsilon$ ), and we consider the four largest values for the tolerance $\varepsilon=0.6$ providing an excessively poor approximation. According to Remark 3.3, we resort to a standard linear interpolation (LIN), a piecewise cubic Hermite (PCH) interpolant, and to an interpolating RBF. Table 3.9 provides the $L^{2}(\Omega)$ - and the $H^{1}(\Omega)$-norm of the relative error associated with the 


\section{ETNA}

Kent State University and

Johann Radon Institute (RICAM)

HIERARCHICAL MODEL REDUCTION DRIVEN BY POD

TABLE 3.8

Directional HiPOD reduction (test case 6): sensitivity to the selected tolerances.

\begin{tabular}{c|c|c|c|c|c|c} 
& Norm & $\varepsilon_{2}=0.6$ & $\varepsilon_{2}=0.9$ & $\varepsilon_{2}=0.99$ & $\varepsilon_{2}=0.999$ & $\varepsilon_{2}=0.9999$ \\
\hline$\varepsilon_{1}=0.6$ & $L^{2}(\Omega)$ & $2.85 \mathrm{e}-01$ & $2.85 \mathrm{e}-01$ & $2.85 \mathrm{e}-01$ & $2.85 \mathrm{e}-01$ & $2.85 \mathrm{e}-01$ \\
& $H^{1}(\Omega)$ & $3.81 \mathrm{e}-01$ & $3.81 \mathrm{e}-01$ & $3.81 \mathrm{e}-01$ & $3.81 \mathrm{e}-01$ & $3.81 \mathrm{e}-01$ \\
\hline$\varepsilon_{1}=0.9$ & $L^{2}(\Omega)$ & $3.27 \mathrm{e}-02$ & $3.27 \mathrm{e}-02$ & $3.06 \mathrm{e}-02$ & $3.06 \mathrm{e}-02$ & $3.06 \mathrm{e}-02$ \\
& $H^{1}(\Omega)$ & $6.49 \mathrm{e}-02$ & $6.49 \mathrm{e}-02$ & $6.45 \mathrm{e}-02$ & $6.45 \mathrm{e}-02$ & $6.45 \mathrm{e}-02$ \\
\hline$\varepsilon_{1}=0.99$ & $L^{2}(\Omega)$ & $1.32 \mathrm{e}-02$ & $1.21 \mathrm{e}-02$ & $3.81 \mathrm{e}-03$ & $3.81 \mathrm{e}-03$ & $3.81 \mathrm{e}-03$ \\
& $H^{1}(\Omega)$ & $1.47 \mathrm{e}-02$ & $1.44 \mathrm{e}-02$ & $1.23 \mathrm{e}-02$ & $1.23 \mathrm{e}-02$ & $1.23 \mathrm{e}-02$ \\
\hline$\varepsilon_{1}=0.999$ & $L^{2}(\Omega)$ & $9.77 \mathrm{e}-03$ & $8.75 \mathrm{e}-03$ & $6.06 \mathrm{e}-04$ & $6.04 \mathrm{e}-04$ & $1.96 \mathrm{e}-04$ \\
& $H^{1}(\Omega)$ & $1.27 \mathrm{e}-02$ & $1.15 \mathrm{e}-02$ & $1.18 \mathrm{e}-03$ & $1.16 \mathrm{e}-03$ & $1.21 \mathrm{e}-03$ \\
\hline$\varepsilon_{1}=0.9999$ & $L^{2}(\Omega)$ & $9.73 \mathrm{e}-03$ & $8.61 \mathrm{e}-03$ & $5.81 \mathrm{e}-04$ & $5.78 \mathrm{e}-04$ & $8.68 \mathrm{e}-05$ \\
& $H^{1}(\Omega)$ & $1.77 \mathrm{e}-02$ & $1.15 \mathrm{e}-02$ & $1.38 \mathrm{e}-03$ & $1.31 \mathrm{e}-03$ & $6.60 \mathrm{e}-04$
\end{tabular}

TABLE 3.9

Directional HiPOD reduction (test case 6): sensitivity to the interpolant operator.

\begin{tabular}{c|c|c|c|c|c} 
& & $\varepsilon=0.9$ & $\varepsilon=0.99$ & $\varepsilon=0.999$ & $\varepsilon=0.9999$ \\
\hline \multirow{2}{*}{ LIN } & $L^{2}(\Omega)$-norm & $7.38 \mathrm{e}-02$ & $1.16 \mathrm{e}-02$ & $4.83 \mathrm{e}-04$ & $6.36 \mathrm{e}-05$ \\
& $H^{1}(\Omega)$-norm & $1.52 \mathrm{e}-01$ & $3.08 \mathrm{e}-02$ & $3.11 \mathrm{e}-03$ & $3.85 \mathrm{e}-04$ \\
\hline \multirow{2}{*}{ PCH } & $L^{2}(\Omega)$-norm & $7.38 \mathrm{e}-02$ & $1.16 \mathrm{e}-02$ & $4.83 \mathrm{e}-04$ & $5.54 \mathrm{e}-05$ \\
& $H^{1}(\Omega)$-norm & $1.52 \mathrm{e}-01$ & $3.08 \mathrm{e}-02$ & $3.11 \mathrm{e}-03$ & $3.84 \mathrm{e}-04$ \\
\hline \multirow{2}{*}{$\mathrm{RBF}$} & $L^{2}(\Omega)$-norm & $7.38 \mathrm{e}-02$ & $1.16 \mathrm{e}-02$ & $4.82 \mathrm{e}-04$ & $5.54 \mathrm{e}-05$ \\
& $H^{1}(\Omega)$-norm & $1.52 \mathrm{e}-01$ & $3.08 \mathrm{e}-02$ & $3.11 \mathrm{e}-03$ & $3.84 \mathrm{e}-04$
\end{tabular}

directional HiPOD approximation with respect to the HiMod solution $u_{20}\left(\alpha^{*}\right)$. For this test case, the $\mathrm{PCH}$ and the RBF interpolants slightly outperform the linear interpolation.

Test case 7. We analyze here the robustness of the directional HiPOD procedure in terms of extrapolation to predict a scenario associated with a value $\alpha^{*}$ of the parameter out of the corresponding range $\mathcal{P}$. For this test, we select as reference configuration the solution to the ADR problem in (2.1)-(2.2) for the set of data

$$
\begin{aligned}
\mu(x, y) & =0.24, \quad \mathbf{b}(x, y)=[5, \sin (6 x)]^{T}, \quad \sigma(x, y)=0, \\
f(x, y) & =10 \chi_{C 1}(x, y)+10 \chi_{C 2}(x, y),
\end{aligned}
$$

with $C_{1}$ and $C_{2}$ defined as in (2.7). From a qualitative viewpoint, the linear finite element approximation to this problem is essentially identical to the solution in Figure 2.2 (top), the reaction $\sigma$ providing a negligible contribution to the solution trend.

The parameter $\alpha$ now coincides with the diffusivity coefficient $\mu$. The offline phase involves the hierarchical reduction of problem (2.1)-(2.2) for ten different values of the viscosity uniformly sampled in the range $\mathcal{P}_{\mu}=[1 / 30,1]$ with all the other problem data being preserved. The HiMod discretization adopted during this stage uses linear finite elements along $\Omega_{1 D}$ in correspondence with a uniform partition of the supporting fiber into 120 subintervals and 20 sinusoidal modes along the transverse direction.

We pick $\alpha^{*}=1 / 60 \notin \mathcal{P}_{\mu}$ as the parameter characterizing the online stage. For comparison purposes, we adopt both the PCH and the RBF interpolations to compute the coefficients $Q_{j}^{k}\left(\alpha^{*}\right)$ in (3.12). This choice is motivated by the higher reliability exhibited, in general, by these two interpolants in terms of extrapolation properties. Table 3.10 compares the rel- 


\section{ETNA}

Kent State University and

Johann Radon Institute (RICAM)

TABLE 3.10

Directional HiPOD reduction (test case 7): robustness to extrapolation.

\begin{tabular}{c|c|c|c|c|c} 
& & $\varepsilon=0.9$ & $\varepsilon=0.99$ & $\varepsilon=0.999$ & $\varepsilon=0.9999$ \\
\hline PCH & $L^{2}(\Omega)$-norm & $2.75 \mathrm{e}-01$ & $1.01 \mathrm{e}-01$ & $9.79 \mathrm{e}-02$ & $9.78 \mathrm{e}-02$ \\
& $H^{1}(\Omega)$-norm & $5.46 \mathrm{e}-01$ & $2.13 \mathrm{e}-01$ & $1.92 \mathrm{e}-01$ & $1.92 \mathrm{e}-01$ \\
\hline \multirow{2}{*}{ RBF } & $L^{2}(\Omega)$-norm & $2.67 \mathrm{e}-01$ & $5.31 \mathrm{e}-02$ & $4.70 \mathrm{e}-02$ & $4.68 \mathrm{e}-02$ \\
& $H^{1}(\Omega)$-norm & $5.36 \mathrm{e}-01$ & $1.44 \mathrm{e}-01$ & $1.02 \mathrm{e}-01$ & $9.97 \mathrm{e}-02$
\end{tabular}
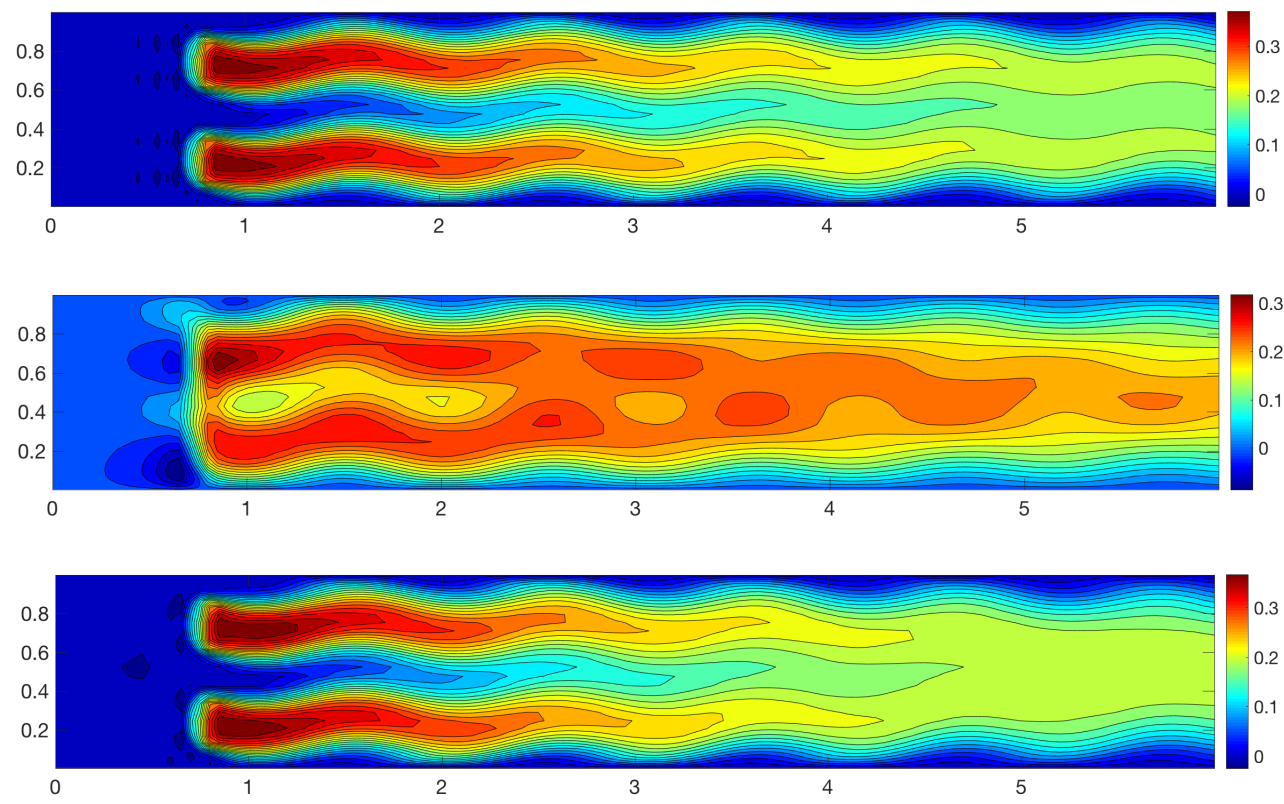

FIG. 3.6. Directional HiPOD reduction (test case 7): robustness to extrapolation. HiMod reference solution (top) and HiPOD approximation for $\varepsilon=0.9$ (center) and $\varepsilon=0.99$ (bottom).

ative modeling error associated with the two interpolants in terms of the $L^{2}(\Omega)$ - and the $H^{1}(\Omega)$-norms. The PCH and RBF procedures are fully comparable with a slightly better performance for the second interpolant. Figure 3.6 provides the contour plots of the reference HiMod solution $u_{20}\left(\alpha^{*}\right)$ and of the directional HiPOD reduction when resorting to the RBF interpolant and for $\varepsilon_{1}=\varepsilon_{2}=\varepsilon=0.9$ and 0.99 (tolerances $\varepsilon=0.999,0.9999$ provide contour plots very similar to the bottom panel). The challenge intrinsic into an extrapolation justifies the large values adopted for the tolerance. The matching between the HiMod and HiPOD approximations is satisfactory.

Test case 8. In this section we extend the HiPOD directional approach to the case when a multiple parameter has to be varied during the offline phase. The use of a vector of parameters leads us to modify the interpolation step of the procedure in Section 3.2. In particular, in order to recover the coefficients $Q_{j}^{k}\left(\boldsymbol{\alpha}^{*}\right)$ in (3.12), we now resort to a two-dimensional interpolant.

As a reference differential setting, we adopt the ADR problem in test case 7, where we identify the parameter with the vector $\boldsymbol{\alpha}=\left[\mu, b_{1}\right]^{T}$, which collects the diffusivity coefficient and the $x$-component of the advective field $\mathbf{b}=\left[b_{1}, b_{2}\right]^{T}$ with the $y$-component being preserved as in (3.14) (i.e., $b_{2}=\sin (6 x)$ ). The set of the admissible parameters is $\mathcal{P}=\mathcal{P}_{\mu} \times \mathcal{P}_{b_{1}}$, with $\mathcal{P}_{\mu}=[1 / 30,1]$ and $\mathcal{P}_{b_{1}}=[0.5,10]$. 
TABLE 3.11

Directional HiPOD reduction (test case 8): relative modeling error for different HiPOD approximations and sensitivity to the interpolant operator for $\boldsymbol{\alpha}_{1}^{*}=[0.6,5.1]^{T}$.

\begin{tabular}{c|c|c|c|c|c|c} 
& & $\varepsilon=0.6$ & $\varepsilon=0.9$ & $\varepsilon=0.99$ & $\varepsilon=0.999$ & $\varepsilon=0.9999$ \\
\hline & $L$ & 2 & 6 & 16 & 32 & 41 \\
& $\max _{j} \mu_{j}$ & 7 & 13 & 19 & 20 & 20 \\
& $\operatorname{median}_{j}$ & 5 & 10 & 16 & 19 & 20 \\
\hline \multirow{2}{*}{ LIN } & $L^{2}(\Omega)$-norm & $1.81 \mathrm{e}-01$ & $5.13 \mathrm{e}-02$ & $3.81 \mathrm{e} 03$ & $6.33 \mathrm{e}-04$ & $5.71 \mathrm{e}-04$ \\
& $H^{1}(\Omega)$-norm & $3.90 \mathrm{e}-01$ & $1.37 \mathrm{e}-01$ & $1.74 \mathrm{e}-02$ & $2.10 \mathrm{e}-03$ & $6.34 \mathrm{e}-04$ \\
\hline \multirow{2}{*}{ PCH } & $L^{2}(\Omega)$-norm & $1.82 \mathrm{e}-01$ & $5.13 \mathrm{e}-02$ & $3.72 \mathrm{e}-03$ & $2.78 \mathrm{e}-04$ & $4.23 \mathrm{e}-05$ \\
& $H^{1}(\Omega)$-norm & $3.90 \mathrm{e}-01$ & $1.37 \mathrm{e}-01$ & $1.74 \mathrm{e}-02$ & $2.01 \mathrm{e}-03$ & $2.51 \mathrm{e}-04$
\end{tabular}

Due to the higher dimensionality of the parameter space, we extend the sampling during the offline phase by hierarchically reducing the reference ADR problem for $p=600$ different choices of the parameter $\boldsymbol{\alpha}$. In particular, the interval $\mathcal{P}_{\mu}$ is sampled with 30 uniformly distributed points, whereas we pick 20 uniformly spaced points along the interval $\mathcal{P}_{b_{1}}$. The HiMod approximation coincides with the one adopted for test case 7, which employs a linear finite element discretization associated with a uniform subdivision of $\Omega_{1 D}$ into 120 subintervals along the mainstream enriched by 20 sinusoidal modal functions to approximate the transverse dynamics. The POD truncation is carried out by identifying the two tolerances in (3.13), and by setting $\varepsilon=\varepsilon_{1}=\varepsilon_{2}=0.6,0.9,0.99,0.999,0.9999$.

The online phase is run to approximate the HiMod solution corresponding to the choices $\boldsymbol{\alpha}_{1}^{*}=[0.6,5.1]^{T}$ and $\boldsymbol{\alpha}_{2}^{*}=[0.06,9.3]^{T}$ for the parameter. Concerning the interpolation step, we adopt both the linear (LIN) and the piecewise cubic Hermite ( $\mathrm{PCH}$ ) bidimensional interpolant operators. In Figures 3.7 and 3.8 we compare the reference HiMod solutions $u_{20}\left(\boldsymbol{\alpha}_{1}^{*}\right)$ and $u_{20}\left(\boldsymbol{\alpha}_{2}^{*}\right)$ with the approximation provided by the directional HiPOD reduction when combined with the PCH interpolation and for the different tolerances. A tolerance sufficiently close to 1 has to be selected to obtain a reliable HiPOD solution. In particular, the choice $\alpha_{2}^{*}$ for the parameter turns out to be more challenging for the HiPOD procedure. This is confirmed also by a cross-comparison between the values in Tables 3.11 and 3.12, which gather the $L^{2}(\Omega)$ - and the $H^{1}(\Omega)$-norms of the relative modeling error associated with the directional HiPOD approximation together with other quantitative data. For the parameter $\boldsymbol{\alpha}_{1}^{*}=[0.6,5.1]^{T}$, it is not immediate to appreciate a remarkable difference between the two interpolants, at least until the tolerance becomes very close to 1 . The PCH interpolation is characterized by a slightly better performance for the parameter $\boldsymbol{\alpha}_{2}^{*}=[0.06,9.3]^{T}$ in particular with respect to the $L^{2}(\Omega)$-norm.

4. Basic versus directional HiPOD approaches. This section has to be understood as an attempt of a comparison between the two HiPOD procedures in Sections 3.1 and 3.2. This task turns out to be not so straightforward due to the strong heterogeneity between the two approaches. The actual goal is to identify specific configurations where one of the two HiPOD methods outperforms the other rather than establishing which is the best formulation ever.

To make the comparison as fair as possible, we test the HiPOD reduction procedures by selecting the same parameters and the corresponding range of variation. In particular:

i) we apply the basic HiPOD approach to the settings in test cases 5 and 6;

ii) we replicate test case 8 with the basic HiPOD approach for the choice $\alpha_{1}^{*}$;

iii) we customize a specific test case which highlights the potentials of the directional method when the two levels of the procedure are properly exploited. 

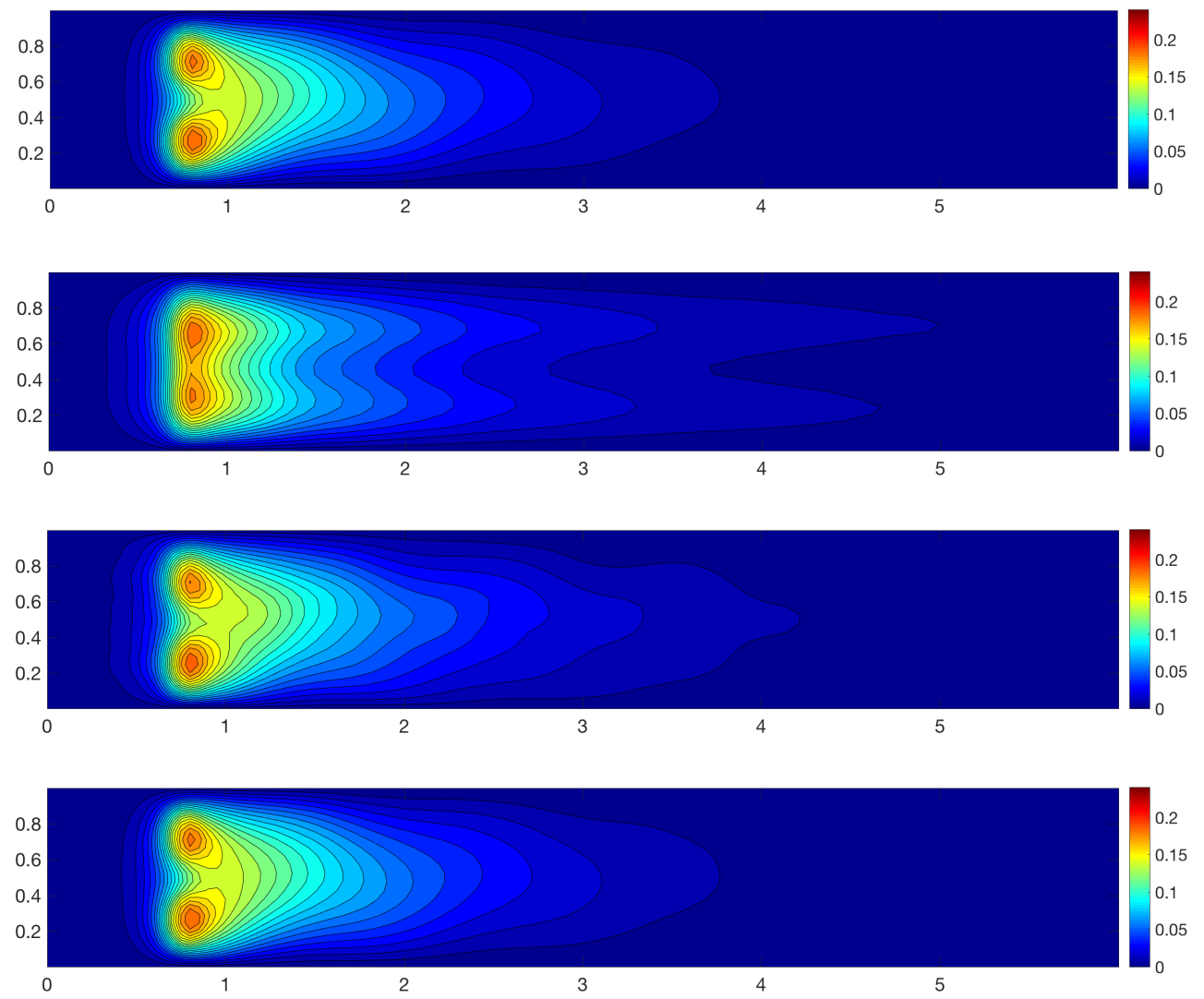

FIG. 3.7. Directional HiPOD reduction (test case 8) for $\boldsymbol{\alpha}_{1}^{*}=[0.6,5.1]^{T}$ : HiMod solution (first row) and HiPOD approximation associated with the PCH interpolant and for $\varepsilon=0.6$ (second row), $\varepsilon=0.9$ (third row), $\varepsilon=0.99$ (fourth row).

i). Test cases 5 and 6 are run with the basic HiPOD reduction procedure to approximate the HiMod solution in Sections 2.1.1 and 2.1.2, respectively. In both cases, we deal with a single parameter setting which identifies $\alpha$ with the viscosity coefficient $\mu$ and with the reactive coefficient $\sigma$, respectively. We exploit the offline phase of the directional approach by computing the HiMod solution for 20 and 30 values of the viscosity and of the reaction uniformly distributed in $\mathcal{P}_{\mu}=[0.15,3]$ and in $\mathcal{P}_{\sigma}=[0.02,0.4]$, respectively. The parameter $\alpha^{*}$ characterizing the online phase is $\alpha^{*}=\mu^{*}=1$ for test case 5 and $\alpha^{*}=\sigma^{*}=0.1$ for test case 6.

Tables 4.1 and 4.2 show the trend of the relative modeling error between the basic HiPOD approximation and the reference HiMod solutions, i.e., $u_{10}\left(\alpha^{*}\right)$ for test case 5 and $u_{20}\left(\alpha^{*}\right)$ for test case 6 , when gradually increasing the dimension $l$ of the POD basis.

The values in Table 4.1 highlight the performance of the basic HIPOD approach, which allows us to gain some order of accuracy with respect to the directional procedure (see Tables 3.4 and 3.5) with a relative small number $(l=10)$ of POD modes. We observe also an unusual increment of both the errors for $l=15$ and $l=17$. This finds a justification in Figure 4.1, which displays the spectrum of the matrix $\mathcal{V}$ in (3.3). Actually, the singular values in $\Sigma$ drop to machine precision around the index 14-15, meaning that the matrix $\mathcal{V}$ is numerically rank deficient. This implies that a POD reduction with a truncated SVD 

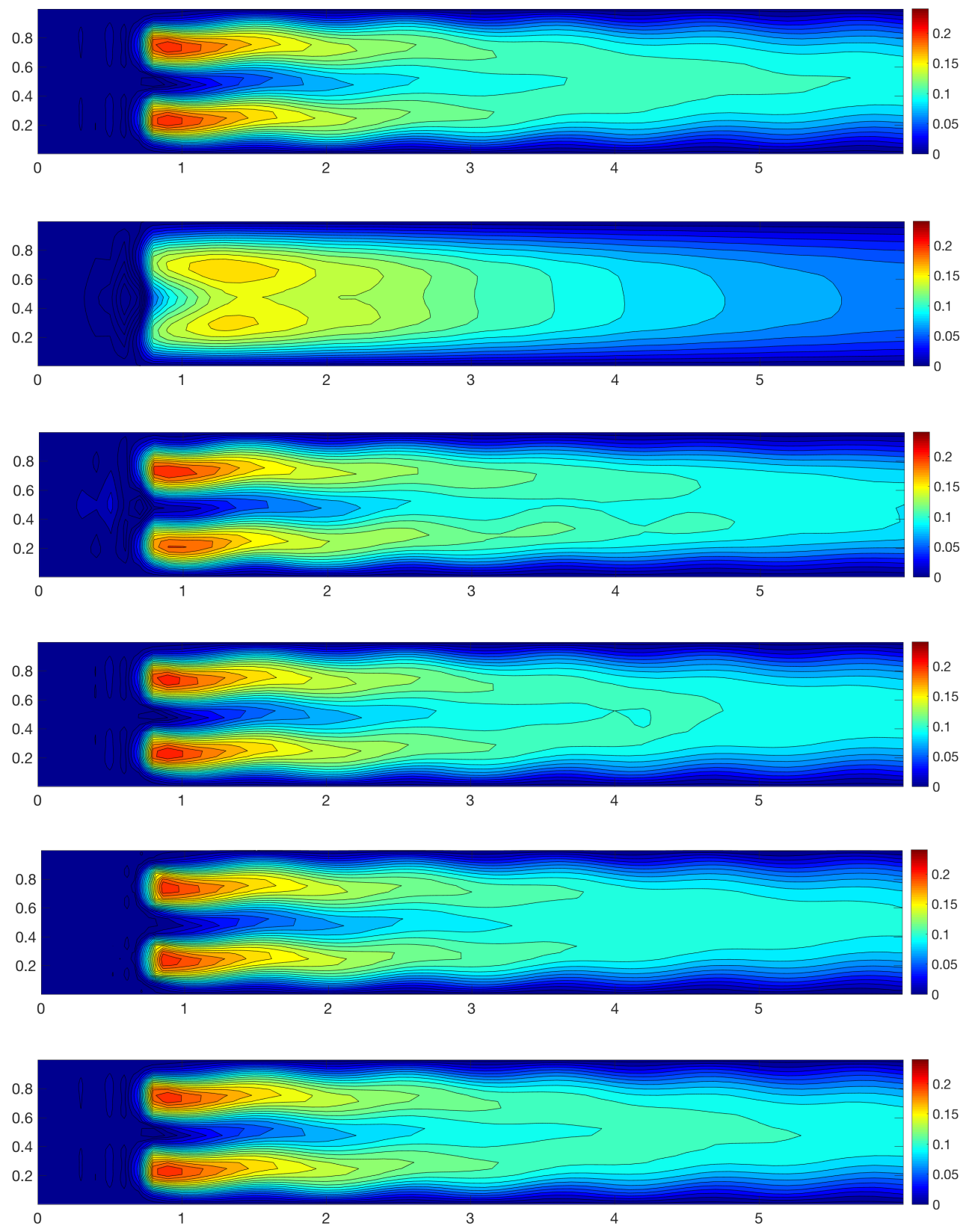

FIG. 3.8. Directional HiPOD reduction (test case 8) for $\boldsymbol{\alpha}_{2}^{*}=[0.06,9.3]^{T}:$ HiMod solution (first row) and HiPOD approximation associated with the PCH interpolant and for $\varepsilon=0.6$ (second row), $\varepsilon=0.9$ (third row), $\varepsilon=0.99$ (fourth row), $\varepsilon=0.999$ (fifth row), and $\varepsilon=0.9999$ (sixth row).

with at most 15 components is enough to capture all the features of the parameter space. Additional components would disadvantage the reconstruction by injecting spurious terms due to numerical instabilities.

As far as Table 4.2 is concerned, we detect the expected decreasing monotonic trend of the error when measured in both the $L^{2}(\Omega)$ - and the $H^{1}(\Omega)$-norm. A cross-comparison with 
TABLE 3.12

Directional HiPOD reduction (test case 8): relative modeling error for different HiPOD approximations and sensitivity to the interpolant operator for $\boldsymbol{\alpha}_{2}^{*}=[0.06,9.3]^{T}$.

\begin{tabular}{c|c|c|c|c|c|c} 
& & $\varepsilon=0.6$ & $\varepsilon=0.9$ & $\varepsilon=0.99$ & $\varepsilon=0.999$ & $\varepsilon=0.9999$ \\
\hline & $L$ & 2 & 6 & 16 & 32 & 41 \\
& $\max _{j} \mu_{j}$ & 7 & 13 & 19 & 20 & 20 \\
& $\operatorname{median}_{j}$ & 5 & 10 & 16 & 19 & 20 \\
\hline \multirow{2}{*}{ LIN } & $L^{2}(\Omega)$-norm & $2.92 \mathrm{e}-01$ & $5.89 \mathrm{e}-02$ & $1.49 \mathrm{e} 02$ & $1.25 \mathrm{e}-02$ & $1.25 \mathrm{e}-02$ \\
& $H^{1}(\Omega)$-norm & $5.34 \mathrm{e}-01$ & $1.73 \mathrm{e}-01$ & $4.48 \mathrm{e}-02$ & $2.76 \mathrm{e}-02$ & $2.76 \mathrm{e}-02$ \\
\hline \multirow{2}{*}{ PCH } & $L^{2}(\Omega)$-norm & $2.93 \mathrm{e}-01$ & $5.76 \mathrm{e}-02$ & $9.22 \mathrm{e}-03$ & $4.30 \mathrm{e}-03$ & $4.14 \mathrm{e}-03$ \\
& $H^{1}(\Omega)$-norm & $5.24 \mathrm{e}-01$ & $1.70 \mathrm{e}-01$ & $3.62 \mathrm{e}-02$ & $1.21 \mathrm{e}-02$ & $9.53 \mathrm{e}-03$
\end{tabular}

TABLE 4.1

Basic HiPOD reduction (test case 5): relative modeling error for different HiPOD approximations.

\begin{tabular}{c|c|c|c|c|c|c} 
& $l=2$ & $l=4$ & $l=7$ & $l=10$ & $l=15$ & $l=17$ \\
\hline$L^{2}(\Omega)$-norm & $1.91 \mathrm{e}-01$ & $1.01 \mathrm{e}-02$ & $9.29 \mathrm{e}-05$ & $2.97 \mathrm{e}-07$ & $9.24 \mathrm{e}-02$ & $1.19 \mathrm{e}-01$ \\
$H^{1}(\Omega)$-norm & $2.11 \mathrm{e}-01$ & $1.14 \mathrm{e}-02$ & $1.19 \mathrm{e}-04$ & $5.22 \mathrm{e}-07$ & $1.58 \mathrm{e}-01$ & $1.89 \mathrm{e}-01$
\end{tabular}

Tables 3.7, 3.8, and 3.9 highlights the fact that the directional HiPOD reduction outperforms the other for this case setting. Finally, the basic and the directional HiPOD procedures are essentially comparable in terms of the computational effort, the wall-clock time being equal to 0.04 and 0.20 seconds for the basic approach to be compared with 0.08 and 0.33 seconds for the directional procedure for the test cases 5 and 6 , respectively.

ii). We move to a multiparameter context by considering test case 8 . We apply the basic HiPOD approach to approximate the HiMod solution associated with the parameter $\boldsymbol{\alpha}_{1}^{*}=\left[\mu^{*}, b_{1}^{*}\right]^{T}=[0.6,5.1]^{T}$. We set up the offline phase as before for the directional procedure based on the HiMod solution for 600 different ADR problems when uniformly varying the parameter $\boldsymbol{\alpha}=\left[\mu, b_{1}\right]^{T}$ in the set of admissible parameters $\mathcal{P}=\mathcal{P}_{\mu} \times \mathcal{P}_{b_{1}}=$ $[1 / 30,1] \times[0.5,10]$. To assess possible benefits of the basic approach in terms of accuracy, we compute the relative modeling error between the basic HiPOD approximation and the HiMod reference solution $u_{20}\left(\alpha_{1}^{*}\right)$ for increasing values of $l$ until all the POD modes are employed (namely, until $l=600$ ). In Table 4.3, we collect the $L^{2}(\Omega)$ - and the $H^{1}(\Omega)$-norm of such an error. A good accuracy is ensured also by the basic HiPOD procedure provided that a sufficiently large number of POD modes is adopted. Nevertheless, the directional approach based on the PCH interpolant allows us to obtain an accuracy improvement by an order of magnitude with respect to the $L^{2}(\Omega)$-norm and without resorting to the full POD spectrum (see Table 3.11).

iii). Some comments on the role played by the two levels in the directional approach are in order to settle the new test case. The singular value decomposition of the matrix $U$ in (3.7) mixes information about the HiMod coefficients at different finite element nodes at the first level. On the other hand, the singular value decomposition of the matrices $S_{j}$ in (3.10) at

TABLE 4.2

Basic HiPOD reduction (test case 6): relative modeling error for different HiPOD approximations.

\begin{tabular}{c|c|c|c|c|c} 
& $l=2$ & $l=4$ & $l=6$ & $l=8$ & $l=10$ \\
\hline$L^{2}(\Omega)$-norm & $7.33 \mathrm{e}-01$ & $5.05 \mathrm{e}-01$ & $7.36 \mathrm{e}-02$ & $8.84 \mathrm{e}-03$ & $7.54 \mathrm{e}-03$ \\
$H^{1}(\Omega)$-norm & $8.33 \mathrm{e}-01$ & $6.72 \mathrm{e}-01$ & $8.09 \mathrm{e}-02$ & $9.84 \mathrm{e}-03$ & $9.34 \mathrm{e}-03$
\end{tabular}




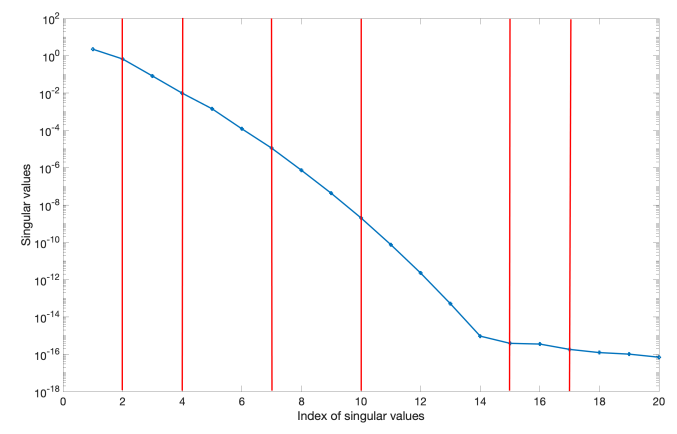

FIG. 4.1. Basic HiPOD reduction (test case 5): singular values of the matrix $\mathcal{V}$.

TABLE 4.3

Basic HiPOD reduction (test case 8): relative modeling error for different HiPOD approximations for $\boldsymbol{\alpha}_{1}^{*}=$ $[0.6,5.1]^{T}$.

\begin{tabular}{c|c|c|c|c|c|c} 
& $l=2$ & $l=10$ & $l=20$ & $l=100$ & $l=400$ & $l=600$ \\
\hline$L^{2}(\Omega)$-norm & $3.01 \mathrm{e}-01$ & $2.22 \mathrm{e}-02$ & $6.41 \mathrm{e}-03$ & $5.80 \mathrm{e}-03$ & $6.60 \mathrm{e}-04$ & $2.43 \mathrm{e}-04$ \\
$H^{1}(\Omega)$-norm & $4.56 \mathrm{e}-01$ & $4.86 \mathrm{e}-02$ & $8.12 \mathrm{e}-03$ & $7.44 \mathrm{e}-03$ & $9.48 \mathrm{e}-04$ & $4.12 \mathrm{e}-04$
\end{tabular}

the second level reveals a possible redundancy of information for the coefficients needed to describe the changes of the HiMod solution over different parameter configurations. Therefore, one would expect that mild changes of the HiMod coefficients across different values of the parameter lead to rank-deficient matrices $S_{j}$. This would translate into a potentially small loss of accuracy when a dimensionality reduction is performed accordingly.

To support this conjecture, we set up a dedicated numerical test. At the $i$ th run of the offline phase, we solve a Poisson problem in the domain $\Omega=\left(0, L_{x}\right) \times\left(0, L_{y}\right)$ completed with homogeneous Dirichlet boundary conditions so that the exact solution is

$$
u_{i}(x, y)=x\left(x-L_{x}\right) \sum_{m=1}^{i} \sin \left(\frac{m \pi y}{L_{y}}\right)
$$

The parameter governing the offline phase is the number $i$ of HiMod modes used to reconstruct the solution $u_{i}$ in exact arithmetic, and, clearly, the complexity of the solution increases with $i$. Because solutions for different problems require a different number of HiMod modes, the accuracy of the HiPOD approximation is expected to be highly sensitive to the performed dimensionality reduction.

Now, we employ the online phase to recover the solution $u_{i^{*}}$ for a random value of the parameter $i$ with $i=i^{*}$ via the directional HiPOD reduction, and we measure the associated (relative) error with respect to both the $L^{2}(\Omega)$ - and the $H^{1}(\Omega)$-norm. Table 4.4 collects the results of such an analysis. The accuracy obtained with the HiPOD approximation is not sensitive to the threshold on the first level, but it is with respect to the threshold at the second level. This is reasonable as the second level retains information about the importance of the HiMod modes in reconstructing the solution and how these modes vary through the parameter space spanned in the offline phase. Moreover, it can be noticed that matrices $S_{j}$ exhibit an upper triangular pattern due to the growing complexity of the solution.

The basic HiPOD reduction is not conceived to work in such a combined way. Actually, by replicating the same test case, it can be verified that the error does not ever decrease below 
TABLE 4.4

Directional HiPOD reduction: relative modeling error for different choices of the tolerances to investigate the role of the two levels.

\begin{tabular}{c|c|c|c|c} 
& & $\varepsilon_{2}=0.6$ & $\varepsilon_{2}=0.9$ & $\varepsilon_{2}=0.99$ \\
\hline$\varepsilon_{1}=0.6$ & $L^{2}(\Omega)$-norm & $8.97 \mathrm{e}+00$ & $2.54 \mathrm{e}+00$ & $3.29 \mathrm{e}-04$ \\
& $H^{1}(\Omega)$-norm & $9.16 \mathrm{e}+01$ & $4.41 \mathrm{e}+01$ & $4.99 \mathrm{e}-02$ \\
\hline \multirow{2}{*}{$\varepsilon_{1}=0.9$} & $L^{2}(\Omega)$-norm & $8.97 \mathrm{e}+00$ & $2.54 \mathrm{e}+00$ & $3.29 \mathrm{e}-04$ \\
& $H^{1}(\Omega)$-norm & $9.16 \mathrm{e}+01$ & $4.41 \mathrm{e}+01$ & $4.99 \mathrm{e}-02$ \\
\hline \multirow{2}{*}{$\varepsilon_{1}=0.99$} & $L^{2}(\Omega)$-norm & $8.97 \mathrm{e}+00$ & $2.54 \mathrm{e}+00$ & $3.29 \mathrm{e}-04$ \\
& $H^{1}(\Omega)$-norm & $9.16 \mathrm{e}+01$ & $4.41 \mathrm{e}+01$ & $4.99 \mathrm{e}-02$
\end{tabular}

5.52e- 01 and $7.25 \mathrm{e}-01$ with respect to the $L^{2}(\Omega)$ - and the $H^{1}(\Omega)$-norm, respectively, even when resorting to all the available POD modes.

5. Conclusions and developments. The numerical assessment in Sections 3.1 .1 and 3.2.1 corroborates the reliability of the HiPOD reduction procedures. We have carried out a more extensive investigation for the directional approach since it represents the main novelty of the paper. In particular, we have analyzed the performances of the directional HiPOD procedure in terms of convergence, the selection of the tolerances driving the truncation of the POD bases, the choice of the interpolant operator, and the robustness with respect to extrapolation. Additionally, the numerical test carried out in the last section seems to suggest that the directional approach outperforms the basic one when dealing with phenomena characterized by a significant horizontal dynamics (i.e., in the considered test cases by an advection field that dominates the diffusivity process).

Despite both the HiPOD procedures deserve a more thorough investigation in 3D and on more generic geometries, we believe that HiPOD model reduction represents a promising tool to effectively manage, for instance, multi-query contexts such as inverse problems, optimization strategies, data assimilation techniques, and parameter estimation algorithms. This makes HiPOD a potential competitor against well-established techniques such as the reduced basis method and the Proper Generalized Decomposition (PGD) (we refer to [27], where a first attempt of a comparison between HiMod/HiPOD reduction and PGD is carried out).

Additionally, we highlight that the techniques here proposed are data-driven approaches so that they do not depend on the specific problem at hand. This could be of great usefulness in view of complex applications. Moreover, HiPOD reduction procedures can be easily generalized by employing any reliable reduced model as the "truth" or by adopting methods other than POD to generate the reduced basis. In such a direction in [39], the authors apply a reduced basis approach to collect the high-fidelity information and use a greedy algorithm to extract the essential information.

As for the possible future research topics, we mention the proposal of rigorous estimators to drive the POD selection $([18,35])$, the generalization of the HiPOD procedures to a nonlinear framework $([5,17,33])$, the application of such techniques to concrete contexts such as in hemodynamic modeling to help clinicians in taking operative decisions [8, 23].

Acknowledgements. This work used resources of the Oak Ridge Leadership Computing Facility (OLCF), which is a DOE Office of Science User Facility supported under Contract DE-AC05-00OR22725.

The second author acknowledges the European Union's Horizon 2020 research and innovation programme under the Marie Skłodowska-Curie Actions, grant agreement 872442 
(ARIA, Accurate Roms for Industrial Applications) and the research project GNCS-INdAM 2020 "Tecniche Numeriche Avanzate per Applicazioni Industriali".

\section{REFERENCES}

[1] M. C. Aletti, S. Perotto, AND A. Veneziani, HiMod reduction of advection-diffusion-reaction problems with general boundary conditions, J. Sci. Comput., 76 (2018), pp. 89-119.

[2] D. AMSALLEM AND C. FARHAT, An online method for interpolating linear parametric reduced-order models, SIAM J. Sci. Comput., 33 (2011), pp. 2169-2198.

[3] C. Audouze, F. De Vuyst, AND P. B. NAIR, Reduced-order modeling of parameterized PDEs using time-space-parameter principal component analysis, Internat. J. Numer. Methods Engrg., 80 (2009), pp. $1025-1057$.

[4] D. Baroli, C. M. Cova, S. Perotto, L. Sala, and A. Veneziani, Hi-POD solution of parametrized fluid dynamics problems: preliminary results, in Model Reduction of Parametrized Systems, P. Benner, M. Ohlberger, A. Patera, G. Rozza, and K. Urban, eds., vol. 17 of MS\&A. Model. Simul. Appl., Springer, Cham, 2017, pp. 235-254.

[5] M. Barrault, Y. Maday, N. C. Nguyen, and A. T. Patera, An 'empirical interpolation' method: application to efficient reduced-basis discretization of partial differential equations, $\mathrm{C}$. R. Math. Acad. Sci. Paris, 339 (2004), pp. 667-672.

[6] U. BAUR, C. BeATtie, P. BENner, AND S. GUGERCin, Interpolatory projection methods for parameterized model reduction, SIAM J. Sci. Comput., 33 (2011), pp. 2489-2518.

[7] P. BENNER, S. GUGERCIN, AND K. WILlCOX, A survey of projection-based model reduction methods for parametric dynamical systems, SIAM Rev., 57 (2015), pp. 483-531.

[8] Y. A. BRANDES COSTA BARBosa AND S. PEROTTO, Hierarchically reduced models for the Stokes problem in patient-specific artery segments, Int. J. Comput. Fluid Dyn., 34 (2020), pp. 160-171.

[9] C. Canuto, Y. Maday, AND A. Quarteroni, Analysis of the combined finite element and Fourier interpolation, Numer. Math., 39 (1982), pp. 205-220.

[10] F. Chinesta, R. Keunings, And A. Leygue, The Proper Generalized Decomposition for Advanced Numerical Simulations: A Primer, Springer, Cham, 2014.

[11] A. ERn And J.-L. Guermond, Theory and Practice of Finite Elements, Springer, New York, 2004

[12] A. ERn, S. PEROTTO, AND A. VENEZIANI, Hierarchical model reduction for advection-diffusion-reaction problems, in Numerical Mathematics and Advanced Applications, K. Kunisch, G. Of, and O. Steinbach, eds., Springer, Berlin, 2008, pp. 703-710.

[13] G. H. Golub and C. F. VAn LoAn, Matrix Computations, 4th ed., Johns Hopkins University Press, Baltimore, 2013

[14] D. González, A. Ammar, F. Chinesta, And E. Cueto, Recent advances on the use of separated representations, Internat. J. Numer. Methods Engrg., 81 (2010), pp. 637-659.

[15] S. GuzZetti, S. Perotto, AND A. Veneziani, Hierarchical model reduction for incompressible fluids in pipes, Internat. J. Numer. Methods Engrg., 114 (2018), pp. 469-500.

[16] B. HEINRICH, The Fourier-finite-element method for Poisson's equation in axisymmetric domains with edges, SIAM J. Numer. Anal., 33 (1996), pp. 1885-1911.

[17] J. S. Hesthaven, G. Rozza, And B. Stamm, Certified Reduced Basis Methods for Parametrized Partial Differential Equations, Springer, Cham, 2016.

[18] M. HINZE AND M. KUnKEL, Residual based sampling in POD model order reduction of drift-diffusion equations in parametrized electrical networks, ZAMM Z. Angew. Math. Mech., 92 (2012), pp. 91-104.

[19] M. KAHLBACHER AND S. VOLKWEIN, Galerkin proper orthogonal decomposition methods for parameter dependent elliptic systems, Discuss. Math. Differ. Incl. Control Optim., 27 (2007), pp. 95-117.

[20] G. Kerschen, J.-C. Golinval, A. F. VAKakis, AND L. A. Bergman, The method of proper orthogonal decomposition for dynamical characterization and order reduction of mechanical systems: an overview, Nonlinear Dynam., 41 (2005), pp. 147-169.

[21] K. KUNISCH AND S. VOLKWEIN, Galerkin proper orthogonal decomposition methods for parabolic problems, Numer. Math., 90 (2001), pp. 117-148.

[22] - Galerkin proper orthogonal decomposition methods for a general equation in fluid dynamics, SIAM J. Numer. Anal., 40 (2002), pp. 492-515.

[23] L. Mansilla Alvarez, P. Blanco, C. Bulant, E. Dari, A. Veneziani, and R. Feijóo, Transversally enriched pipe element method (TEPEM): an effective numerical approach for blood flow modeling, Int. J. Numer. Methods Biomed. Eng., 33 (2017), Art. e02808, 24 pages.

[24] M. OHLBERGER AND K. SMETANA, Approximation of skewed interfaces with tensor-based model reduction procedures: application to the reduced basis hierarchical model reduction approach, J. Comput. Phys., 321 (2016), pp. 1185-1205.

[25] S. PERotTo, Hierarchical model (Hi-Mod) reduction in non-rectilinear domains, in Domain Decomposition 


\section{ETNA}

Kent State University and

Johann Radon Institute (RICAM)

Methods in Science and Engineering XXI, J- Erhel, M. J. Gander, L. Halpern, G. Pichot, T. Sassi, and O. Widlund, eds., vol. 98 of Lect. Notes Comput. Sci. Eng., Springer, Cham, 2014, pp. 477-485.

[26] _ - A survey of hierarchical model (Hi-Mod) reduction methods for elliptic problems, in Numerical Simulations of Coupled Problems in Engineering, S. R. Idelsohn, ed., vol. 33 of Comput. Methods Appl. Sci., Springer, Cham, 2014, pp. 217-241.

[27] S. Perotto, M. G. CARLINO, AND F. BALlaRin, Model reduction by separation of variables: a comparison between hierarchical model reduction and proper generalized decomposition, in Spectral and High Order Methods for Partial Differential Equations-ICOSAHOM 2018, S. J. Sherwin, D. Moxey, J. Peiró, P. E. Vincent, and C. Schwab, eds., vol. 134 of Lect. Notes Comput. Sci. Eng., Springer, Cham, 2020, pp. 61-77.

[28] S. Perotto, A. ERn, And A. Veneziani, Hierarchical local model reduction for elliptic problems: a domain decomposition approach, Multiscale Model. Simul., 8 (2010), pp. 1102-1127.

[29] S. Perotto, A. Reali, P. Rusconi, AND A. Veneziani, HIGAMod: a hierarchical isogeometric approach for model reduction in curved pipes, Comput. \& Fluids, 142 (2017), pp. 21-29.

[30] S. Perotto AND A. Veneziani, Coupled model and grid adaptivity in hierarchical reduction of elliptic problems, J. Sci. Comput., 60 (2014), pp. 505-536.

[31] S. PEROTTO AND A. ZiLIO, Hierarchical model reduction: three different approaches, in Numerical Mathematics and Advanced Applications, A. Cangiani, R. L. Davidchack, E. Georgoulis, A. N. Gorban, J. Levesley, and M. V. Tretyakov, eds., 2011, Springer, Heidelberg, 2013, pp. 851-859.

[32] _ - Space-time adaptive hierarchical model reduction for parabolic equations, Adv. Model. and Simul. Eng. Sci., (2015), Art. 25, 45 pages.

[33] A. Quarteroni, A. Manzoni, And F. Negri, Reduced Basis Methods for Partial Differential Equations, Springer, Cham, 2016.

[34] M. VogeliUS AND I. BABUŠKA, On a dimensional reduction method. I. The optimal selection of basis functions, Math. Comp., 37 (1981), pp. 31-46.

[35] S. VOLKWEIN, Optimality system POD and a-posteriori error analysis for linear-quadratic problems, Control Cybernet., 40 (2011), pp. 1109-1124.

[36] S. Volkwein, Proper Orthogonal Decomposition: Theory and Reduced-Order Modelling, Lecture Notes, University of Konstanz, Konstanz, 2013.

[37] S. WALTON, O. HASSAN, AND K. MORGAN, Reduced order modelling for unsteady fluid flow using proper orthogonal decomposition and radial basis functions, Appl. Math. Model., 37 (2013), pp. 8930-8945.

[38] H. WendLAND, Piecewise polynomial, positive definite and compactly supported radial functions of minimal degree, Adv. Comput. Math., 4 (1995), pp. 389-396.

[39] M. Zancanaro, F. Ballarin, S. Perotto, And G. Rozza, Hierarchical model reduction techniques for flow modeling in a parametrized setting, Multiscale Model. Simul., 19 (2021), pp. 267-293. 